\title{
The Mc- $\Gamma$ Distribution and Its Statistical Properties: An Application to Reliability Data
}

\author{
Francisco W. P. Marciano (Corresponding author) \\ Departamento de Estatística, Universidade Federal de Pernambuco \\ Cidade Universitária, 50740-540, Recife, PE, Brazil
}

Tel: 55-81-2126-7434 E-mail: bill.marciano@gmail.com

A. D. C. Nascimento

Departamento de Estatística, Universidade Federal de Pernambuco

Cidade Universitária, 50740-540, Recife, PE, Brazil

Tel: 55-81-2126-7434Ｅ-mail: abraao.susej@gmail.com

\section{Santos-Neto (Note 1)}

Departamento de Matemática e Estatística, Universidade Federal de Campina Grande Av. Aprígio Veloso, 882, Bloco CX, 58.109-970, Bodocongó, Campina Grande, PB, Brazil

Tel: 55-83-2101-1637 E-mail: santosneto@dme.ufcg.edu.br

\section{G. M. Cordeiro}

Departamento de Estatística, Universidade Federal de Pernambuco

Cidade Universitária, 50740-540, Recife, PE, Brazil

Tel: 55-81-2126-7434Ｅ-mail: gauss@de.ufpe.br

Received: February 15, 2012 Accepted: March 5, 2012 Published: May 1, 2012

doi:10.5539/ijsp.v1n1p53 URL: http://dx.doi.org/10.5539/ijsp.v1n1p53

\begin{abstract}
The gamma distribution has been widely used in many research areas such as engineering, hydrology and survival analysis. We propose a new distribution, called the McDonald gamma distribution, which presents greater flexibility to model scenarios involving non-negative data. The new density function is represented as a double linear combination of gamma densities. We also propose analytical expressions for some mathematical quantities: moments, moment generating function, log-moment, mean deviations, Lorentz and Bonferroni curves, order statistics, entropy and quantile function. The score function and the observed information matrix of this new distribution are derived. A real data set is used to illustrate the importance of the proposed model.
\end{abstract}

Keywords: Beta-Generated class, Entropy, Generalized distribution, Maximum likelihood estimation, Moment

\section{Introduction}

The gamma distribution is a very general distribution that belongs to the Pearson type III family of distributions. It includes, among other well-known distributions, the exponential and chi-square distributions. Some of its structural properties can be found in Jambunathan (1954). It has a variety of applications and can be used to model the queuing systems, the flow of items through manufacturing and distribution processes, the risk management and some distributions in hydrology. More detailed information on hydrology can be found in (Yevjevich, 1972; Bobee \& Ashkar, 1991).

Several generalized distributions have been studied in recent years. The generalization of continuous distributions began with Amoroso (1925). He introduced the generalized gamma (GG) distribution for income rate data. It is also discussed by Stacy (1962). Esteban (1981) showed that the GG distribution has several distributions as special cases or limiting forms, for example, the log-normal, Weibull, gamma, exponential, normal and Pareto distributions. The GG distribution has several applications in areas such as engineering, hydrology and survival analysis, and it is very useful in discriminating between alternative probabilistic models. Nadarajah \& Gupta (2007) applied it to drought data. Nadarajah (2008) 
presented a study on its use in electrical and electronic engineering. Cox (2008) studied the F-generalized family by comparing it with the GG model. Cordeiro et al. (2011) proposed the exponentiated GG distribution with application to lifetime data and Pascoa et al. (2011) defined the called Kumaraswamy GG distribution with application in survival analysis, since it is capable of modeling a bathtub-shaped hazard rate function.

In recent years, several authors published new distributions. Eugene et al. (2002) introduced a class of generalized distributions based on the logit of the beta random variable. They proposed the beta normal distribution. After their work, new distributions have been developed in this class, such as the beta Fréchet (Nadarajah \& Gupta, 2004), beta Gumbel (Nadarajah \& Kotz, 2004), beta exponential (Nadarajah \& kotz, 2005), beta Weibull (Lee et al., 2007), beta Pareto (Akinsete et al., 2008), beta half-normal (Pescim et al., 2006), beta generalized exponential (Barreto-Souza et al., 2010) and beta power (Cordeiro \& Brito, 2012) distributions.

In this sense, our purpose is to present a new distribution, called the McDonald gamma distribution (Mc- $\Gamma$ for short), which extends the gamma model and has several other models as special cases. Some of its mathematical properties are obtained and the method of maximum likelihood estimation is discussed. The new model provides greater flexibility than other distributions, since it has more shape parameters, yielding a large variety of forms. It can also be useful for testing the goodness of fit of its sub-models.

The rest of the article is organized as follows. In Section 2, we discuss the modeling of the Mc- $\Gamma$ distribution, in which the class $\mathrm{Mc}$ is contextualized. We also present its density function, cumulative distribution and hazard function. Some expansions of its mathematical quantities are derived in Section 3. Additionally, its moment generating function (mgf) and the limiting density and cumulative distribution functions are derived. Moreover, we propose analytical expressions for the following statistical measures: Shannon and Rényi entropies, mean deviations, Bonferroni and Lorentz curves, skewness, kurtosis, order statistics and quantile function. In Section 4, we discuss maximum likelihood estimation. In Section 5, the Mc-Г distribution is applied to a real data set. Finally, in Section 6, we provide some concluding remarks.

\section{The Mc- $\Gamma$ Distribution}

The Mc- $\Gamma$ distribution originated from the work of Eugene et al. (2002) who defined a general class of distributions as follows: if $\mathrm{G}$ denotes the cumulative distribution function (cdf) of a random variable, then a generalized class of distributions can be defined as

$$
F(x)=I_{G(x)}(a, b)=\frac{1}{B(a, b)} \int_{0}^{G(x)} \omega^{a-1}(1-\omega)^{b-1} \mathrm{~d} \omega,
$$

for $a>0, b>0$, where $I_{y}(a, b)=B_{y}(a, b) / B(a, b)$ denotes the incomplete beta function ratio and $B_{y}(a, b)=\int_{0}^{y} \omega^{a-1}(1-$ $\omega)^{b-1} \mathrm{~d} \omega$ is the incomplete beta function. The probability density function (pdf) corresponding to (1) can be expressed as

$$
f(x)=\frac{1}{B(a, b)} G(x)^{a-1}[1-G(x)]^{b-1} g(x),
$$

where $g(x)=\partial G(x) / \partial x$ is the baseline density function.

We start with the generalized beta distribution of the first kind (or beta type I) introduced by McDonald (1984). Its pdf is given by

$$
f(x)=\frac{c}{B\left(\frac{a}{c}, b\right)} x^{a / c-1}\left(1-x^{c}\right)^{b-1},
$$

where $a>0, b>0$ and $c>0$ are shape parameters.

Alexander et al. (2011) introduce the generalized beta-generated (GBG) distribution which has as sub-models the classical beta-generated, Kumaraswamy-generated and exponentiated distributions. Consider starting from an arbitrary baseline continuous distribution function $G(x)$, the $\operatorname{cdf} F(x)$ of the GBG distribution can be expressed as

$$
F(x)=I_{G(x)^{c}}\left(\frac{a}{c}, b\right)=\frac{1}{B\left(\frac{a}{c}, b\right)} \int_{0}^{G(x)^{c}} \omega^{a / c-1}(1-\omega)^{b-1} \mathrm{~d} \omega .
$$

We follow the works of Eugene et al. (2002), Jones (2004), Cordeiro \& de Castro (2011) and Alexander et al. (2011) to define the Mc- $\Gamma$ distribution. If we substitute the gamma cdf in (2), we obtain the $\mathrm{Mc}-\Gamma$ cumulative distribution.

Here and henceforth, consider the pdf of the $\Gamma(\alpha, \beta)$ distribution given by

$$
h(x ; \alpha, \beta)=\frac{\beta^{\alpha}}{\Gamma(\alpha)} x^{\alpha-1} \exp (-\beta x), \quad x>0,
$$


where $\alpha>0$ and $\beta>0$ are shape and scale parameters, respectively.

Thus, the Mc- $\Gamma$ cumulative distribution is

$$
F_{\mathrm{Mc}-\Gamma}(x ; \alpha, \beta, a, b, c)=\frac{1}{B\left(\frac{a}{c}, b\right)} \int_{0}^{\gamma_{1}(\alpha, \beta x)^{c}} w^{\frac{a}{c}-1}(1-w)^{b-1} \mathrm{~d} w,
$$

where $\gamma_{1}(\alpha, \beta x)=\frac{1}{\Gamma(\alpha)} \int_{0}^{\beta x} t^{\alpha-1} \mathrm{e}^{-t} \mathrm{~d} t$ is the incomplete gamma function ratio, $\beta>0$ is a scale parameter and $\alpha, a, b, c>0$ are shape parameters.

The Mc- $\Gamma$ density function is obtained by differentiating (3). We have

$$
f_{\mathrm{Mc}-\Gamma}(x ; \alpha, \beta, a, b, c)=\frac{c \beta^{\alpha} x^{\alpha-1} \mathrm{e}^{-\beta x}}{\Gamma(\alpha) B\left(\frac{a}{c}, b\right)} \gamma_{1}(\alpha, \beta x)^{a-1}\left[1-\gamma_{1}(\alpha, \beta x)^{c}\right]^{b-1} \text {. }
$$

If $X$ is a random variable with density (4), we write $X \sim \operatorname{Mc}-\Gamma(\alpha, \beta, a, b, c)$. Two important special sub-models are the beta gamma $(\mathcal{B}-\Gamma)$ distribution (when $c=1$ ), proposed by Jones (2004), and the Kumaraswamy gamma $(\mathrm{Kw}-\Gamma)$ distribution (when $a=1$ ), studied by Cordeiro \& de Castro (2011). The Mc- $\Gamma$ density function for selected parameter values is plotted in Figs. 1(a)-1(b).

The survival and hazard rate function of the $\mathrm{Mc}-\Gamma$ distribution are

$$
S_{\mathrm{Mc}-\Gamma}(x ; \alpha, \beta, a, b, c)=1-\frac{1}{B\left(\frac{a}{c}, b\right)} \int_{0}^{\gamma_{1}(\alpha, \beta x)^{c}} w^{\frac{a}{c}-1}(1-w)^{b-1} \mathrm{~d} w
$$

and

respectively.

$$
h_{\mathrm{Mc}-\Gamma}(x ; \alpha, \beta, a, b, c)=\frac{c \beta^{\alpha} x^{\alpha-1} \mathrm{e}^{-\beta x} \gamma_{1}(\alpha, \beta x)^{a-1}\left[1-\gamma_{1}(\alpha, \beta x)^{c}\right]^{b-1}}{\Gamma(\alpha) B\left(\frac{a}{c}, b\right) S_{\mathrm{Mc}-\Gamma}(x ; \alpha, \beta, a, b, c)},
$$

In Figs. 2(a)-2(b), we plot the hazard function for selected parameter values. This function is quite flexible and may take different forms: constant, increasing, decreasing and bathtub.

To generate random numbers from the $\operatorname{Mc}-\Gamma(\alpha, \beta, a, b, c)$ distribution, we have to solve the nonlinear equation

$$
\gamma_{1}(\alpha, \beta X)-U^{1 / c}=0,
$$

where $U \sim \mathcal{B}\left(\frac{a}{c}, b\right)$. We use the R programming language ( $\mathrm{R}$ Development Core Team, 2008) for solving this equation. Figs. 3(a)-3(b). present the theoretical and approximate densities for different sample sizes, $n \in\{500,2000,10000\}$. In Fig. 3(b), we provide the histogram of the simulated data for $n=500$. Clearly, the data are well-fitted by the Mc- $\Gamma$ theoretical density.

\subsection{Special Sub-models}

The Mc- $\Gamma$ distribution contains as special cases several well-known distributions shown in Fig. 4. In addition to these distributions, it contains other special cases such as: $\operatorname{Mc}-\chi^{2}(\alpha=k / 2, \beta=1 / 2), \operatorname{Mc}-\operatorname{Exp}(\alpha=1), \mathrm{Kw}-\chi^{2}(\alpha=k / 2, \beta=$ $1 / 2, a=1), \mathrm{Kw}-\operatorname{Exp}(\alpha=1, a=1), \mathcal{B}-\chi^{2}(\alpha=k / 2, \beta=1 / 2, c=1), \mathcal{B}-\operatorname{Exp}(\alpha=1, c=1), \mathcal{L}_{1}-\chi^{2}(\alpha=k / 2, \beta=1 / 2, b=$ 1), $\mathcal{L}_{1}-\operatorname{Exp}(\alpha=1, b=1), \mathcal{L}_{2}-\chi^{2}(\alpha=k / 2, \beta=1 / 2, a=1, c=1), \mathcal{L}_{2}$-Exp $(\alpha=1, a=1, c=1)$. Here, Kw-G denotes the family of Kumaraswamy $\mathrm{G}$ distributions, $\mathcal{B}-\mathrm{G}$ the family of beta $\mathrm{G}$ distributions, $\mathcal{L}_{1}-\mathrm{G}$ the family of Lehmann type I $\mathrm{G}$ distributions and $\mathcal{L}_{2}-\mathrm{G}$ the family of Lehmann type II $\mathrm{G}$ distributions.

\section{Theoretical Properties}

\subsection{Expansions for Important Mathematical Quantities}

Theorem 1. Here and henceforth, let $X \sim M c-\Gamma(\alpha, \beta, a, b, c)$. If $s$ is a non-negative real number, we obtain the double linear combination

$$
f_{M c-\Gamma}^{s}(x ; \alpha, \beta, a, b, c)=\sum_{v, m=0}^{\infty} w_{v, m}^{(s)} h(x ; \alpha v+m+1+s(\alpha-1), \beta),
$$

where $h(x ; \alpha v+m+1+s(\alpha-1), \beta)$ is the gamma density with shape parameter $\alpha v+m+1+s(\alpha-1)$ and scale parameter $\beta$,

$$
w_{v, m}^{(s)}=\frac{t_{m, v} \Gamma(\alpha v+m+1+s(\alpha-1))}{\beta^{m+1-s} \Gamma(\alpha)^{v} \sum_{i, k=0}^{\infty}\left(\begin{array}{c}
i c+s(a-1) \\
k
\end{array}\right)\left(\begin{array}{c}
s(b-1) \\
i
\end{array}\right)\left(\begin{array}{l}
k \\
v
\end{array}\right)(-1)^{i+k+v}},
$$

and the quantity $t_{m, v}$ is defined in Appendix A. 
The proof of this theorem is given in Appendix A.

Corollary 1. We obtain the double linear combination for the density function of $X$

$$
f_{M c-\Gamma}(x ; \alpha, \beta, a, b, c)=\sum_{v, m=0}^{\infty} w_{v, m}^{(1)} h(x ; \alpha v+m+\alpha, \beta),
$$

where $w_{v, m}^{(1)}$ is given in Theorem 1.

Corollary 2. If $Y \sim \Gamma(\alpha v+m+\alpha, \beta)$, explicit expressions for the cdf, nth moment and mgf of $X$ are given by

$$
\begin{aligned}
F_{M c-\Gamma}(x ; \alpha, \beta, a, b, c) & =\sum_{v, m=0}^{\infty} w_{v, m}^{(1)} \gamma_{1}(\alpha v+m+\alpha, \beta), \\
\mathrm{E}\left(X^{n}\right) & =\sum_{v, m=0}^{\infty} w_{v, m}^{(1)} \mathrm{E}\left(Y^{n}\right)=\sum_{v, m=0}^{\infty} w_{v, m}^{(1)} \frac{\Gamma(\alpha v+m+\alpha+n)}{\Gamma(\alpha v+m+\alpha) \beta^{n}}, \\
\mathrm{M}_{X}(t) & =\sum_{v, m=0}^{\infty} w_{v, m}^{(1)} \mathrm{M}_{Y}(t)=\sum_{v, m=0}^{\infty} w_{v, m}^{(1)}(1-\beta t)^{-(\alpha v+m+\alpha)},
\end{aligned}
$$

for $t<\beta$, respectively, where $w_{v, m}^{(1)}$ is given in Theorem 1 .

\subsection{Asymptotic Density and Cumulate Distribution Functions}

Consider the representation in power series in Appendix B and the asymptotic results for the exponentiated gamma density function given by Nadarajah and Kotz (2006). The following approximations for the asymptotic density and cumulate distribution functions hold:

$$
f_{\mathrm{Mc}-\Gamma}(x ; \alpha, \beta, a, b, c) \sim\left\{\begin{array}{l}
\sum_{k=1}^{\infty} w_{k}^{\prime}\left\{\frac{(c k+1) \alpha^{-c k} x^{\alpha c k}}{\Gamma(\alpha)^{c k+1}}\right\}, x \rightarrow 0, \\
\sum_{k=1}^{\infty} w_{k}^{\prime}\left\{\frac{(c k+1) x^{\alpha-1} \exp (-x)}{\Gamma(c k+1)}\right\}, x \rightarrow \infty
\end{array}\right.
$$

and

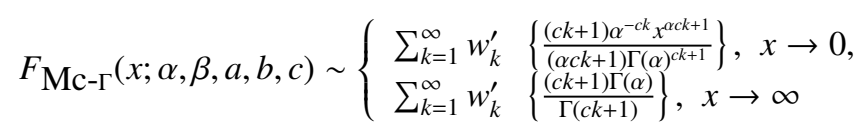

where

$$
w_{k}^{\prime}=\frac{c \beta(-1)^{k}\left(\begin{array}{c}
b-1 \\
k
\end{array}\right)}{(c k+1) B\left(\frac{a}{c}, b\right)}
$$

\subsection{Alternative Forms for Ordinary Moments}

The following discussion proposes an alternative expression for the $n t h$ ordinary moment of $X$. We have

$$
\mathrm{E}\left(X^{n}\right)=\frac{c \beta}{\Gamma(\alpha) B\left(\frac{a}{c}, b\right)} \int_{0}^{\infty} x^{n+\alpha-1} \mathrm{e}^{-\beta x}\left[\gamma_{1}(\alpha, \beta x)\right]^{a-1}\left[1-\gamma_{1}(\alpha, \beta x)^{c}\right]^{b-1} \mathrm{~d} x
$$

Since $\gamma_{1}(\alpha, \beta x)<1$ and $c>0$, the following expansion holds

$$
\left[1-\gamma_{1}(\alpha, \beta x)^{c}\right]^{b-1}=\sum_{i=0}^{\infty}(-1)^{i}\left(\begin{array}{c}
b-1 \\
i
\end{array}\right) \gamma_{1}(\alpha, \beta x)^{c i}
$$

Additionally, we work with an expansion for the incomplete gamma function

$$
\gamma_{1}(\alpha, \beta x)=\frac{(\beta x)^{\alpha}}{\Gamma(\alpha)} \sum_{m=0}^{\infty} \frac{(-\beta x)^{m}}{(\alpha+m) m !} .
$$


Thus, equation (6) can be reduced to

$$
\begin{aligned}
\mathrm{E}\left(X^{n}\right) & =\frac{c \beta^{\alpha}}{\Gamma(\alpha) B\left(\frac{a}{c}, b\right)} \sum_{i=0}^{\infty}(-1)^{i}\left(\begin{array}{c}
b-1 \\
i
\end{array}\right) \int_{0}^{\infty} x^{n+\alpha-1} \mathrm{e}^{-\beta x} \gamma_{1}(\alpha, \beta x)^{i c+a-1} \mathrm{~d} x \\
& =\frac{c \beta^{\alpha}}{\Gamma(\alpha) B\left(\frac{a}{c}, b\right)} \sum_{i=0}^{\infty} \frac{(-1)^{i}}{\Gamma(\alpha)^{i c+a-1}}\left(\begin{array}{c}
b-1 \\
i
\end{array}\right) \int_{0}^{\infty} x^{n+\alpha-1} \mathrm{e}^{-\beta x}\left\{(\beta x)^{\alpha} \sum_{m=0}^{\infty} \frac{(-\beta x)^{m}}{(\alpha+m) m !}\right\}^{i c+a-1} \mathrm{~d} x .
\end{aligned}
$$

Setting $u=\beta x$, we have

$$
\begin{aligned}
\mathrm{E}\left(X^{n}\right) & =\frac{c \beta^{-n}}{\Gamma(\alpha) B\left(\frac{a}{c}, b\right)} \sum_{i=0}^{\infty} \frac{(-1)^{i}}{\Gamma(\alpha)^{i c+a-1}}\left(\begin{array}{c}
b-1 \\
i
\end{array}\right) \underbrace{\int_{0}^{\infty} u^{n+\alpha-1} \exp (-u)\left\{u^{\alpha} \sum_{m=0}^{\infty} \frac{(-u)^{m}}{(\alpha+m) m !}\right\}^{i c+a-1} \mathrm{~d} u}_{\text {Lauricella function of type } \mathrm{A} \text { (Cordeiro \& Nadarajah, 2011) }} . \\
& =\frac{c \beta^{-n}}{\Gamma(\alpha) B\left(\frac{a}{c}, b\right)} \sum_{i=0}^{\infty} \frac{(-1)^{i} \alpha^{-(i c+a-1)}}{\Gamma(\alpha)^{i c+a-1}}\left(\begin{array}{c}
b-1 \\
i
\end{array}\right) \Gamma(n+\alpha(i c+a)) F_{A}^{(i c+a-1)}(n+\alpha(i c+a) ; \alpha, \ldots, \alpha ; \alpha+1, \ldots, \alpha+1 ;-1, \ldots,-1) .
\end{aligned}
$$

\subsection{Expression for the Rényi Entropy}

Let $Y$ be a random variable with density $f(y ; \theta)$ and support $y \in \mathcal{D} \subset \mathbb{R}$. The Rényi entropy is defined by

$$
H_{\mathrm{R}}^{s}(Y)=\frac{1}{1-s} \log \left\{\mathrm{E}\left[f(y ; \theta)^{s-1}\right]\right\}=\frac{1}{1-s} \log \left(\int_{\mathcal{D}} f(y ; \theta)^{s} \mathrm{~d} y\right),
$$

where $s \geq 0$ and $s \neq 1$.

From the expansion given in Theorem 1, we can write

$$
\begin{aligned}
H_{\mathrm{R}}^{s}(X)= & \frac{1}{1-s} \log \left(\sum_{v, m=0}^{\infty} w_{v, m}^{(s)} \int_{0}^{\infty} h(x ; \alpha \nu+m+1+s(\alpha-1), \beta) \mathrm{d} x\right) \\
& =\frac{1}{1-s} \log \left(\sum_{v, m=0}^{\infty} w_{v, m}^{(s)}\right),
\end{aligned}
$$

where $w_{v, m}^{(s)}$ is given in Theorem 1 .

\subsection{Expressions for the Log-moment and Shannon Entropy}

Let $Y$ be defined as in Section 3.4. The log-moment is given by

$$
\mathrm{E}\left\{[\log (Y)]^{n}\right\}=\int_{\mathcal{D}} \log (y)^{n} f(y ; \theta) \mathrm{d} y .
$$

Now, we consider $Y \sim \Gamma(\alpha, \beta)$ with density function $h(x ; \alpha, \beta)$. Minor manipulations yield

$$
\mathrm{E}\left\{[\log (Y)]^{n}\right\}=\frac{\beta^{\alpha}}{\Gamma(\alpha)}\left\{\frac{\partial^{(n)}}{\partial \alpha^{n}}\left(\frac{\Gamma(\alpha)}{\beta^{\alpha}}\right)\right\} \stackrel{n=1}{\longrightarrow} \mathrm{E}[\log (Y)]=\psi(\alpha)-\log (\beta),
$$

where $\psi(\cdot)$ is the digamma function.

Combining this result with Corollary 1 , if $Z \sim \Gamma(\alpha v+m+\alpha, \beta)$, we have

$$
\begin{aligned}
\mathrm{E}\left\{[\log (X)]^{n}\right\} & =\sum_{v, m=0}^{\infty} w_{v, m}^{(1)} \mathrm{E}\left\{[\log (Z)]^{n}\right\} \\
& =\sum_{v, m=0}^{\infty} w_{v, m}^{(1)} \frac{\beta^{\alpha v+m+\alpha}}{\Gamma(\alpha v+m+\alpha)}\left\{\frac{\partial^{(n)}}{\partial j^{n}}\left(\frac{\Gamma(j)}{\beta^{j}}\right)\right\}_{j=\alpha v+m+\alpha} \\
& \stackrel{n=1}{\longrightarrow} \\
\mathrm{E}[\log (X)] & =\sum_{v, m=0}^{\infty} w_{v, m}^{(1)}\{\psi(\alpha v+m+\alpha)-\log (\beta)\}
\end{aligned}
$$


In the following discussion, we derive the Shannon entropy defined by

$$
H_{\mathrm{S}}(X)=\mathrm{E}\left\{-\log \left[f_{\mathrm{Mc}-\Gamma}(X)\right]\right\}=\int_{0}^{\infty}-\log \left[f_{\mathrm{Mc}-\Gamma}(x)\right] f_{\mathrm{Mc}-\Gamma}(x) \mathrm{d} x .
$$

The log-likelihood function relative to one observation follows from (4) as

$$
\ell(\alpha, \beta, a, b, c ; x)=\log \left[\frac{c \beta^{\alpha}}{\Gamma(\alpha) B\left(\frac{a}{c}, b\right)}\right]+(\alpha-1) \log (x)-\beta x+(a-1) \log \left[\gamma_{1}(\alpha, \beta x)\right]+(b-1) \log \left[1-\gamma_{1}(\alpha, \beta x)^{c}\right] .
$$

It is known that the expected value of the score function vanishes, and then from $\mathrm{E}\{\partial \ell(\theta) / \partial a\}=0$ and $\mathrm{E}\{\partial \ell(\theta) / \partial b\}=0$, we obtain

$$
\mathrm{E}\left\{\log \left[\gamma_{1}(\alpha, \beta X)\right]\right\}=\frac{\psi\left(\frac{a}{c}\right)-\psi\left(\frac{a}{c}+b\right)}{c}
$$

and

$$
\mathrm{E}\left\{\log \left[1-\gamma_{1}(\alpha, \beta X)^{c}\right]\right\}=\psi(b)-\psi\left(\frac{a}{c}+b\right),
$$

respectively.

Hence, the Shannon entropy of the Mc- $\Gamma$ distribution reduces to

$$
\begin{aligned}
H_{\mathrm{S}}(X)= & \log \left(\frac{\Gamma(\alpha) B\left(\frac{a}{c}, b\right)}{c \beta^{\alpha}}\right)-(\alpha-1) \sum_{v, m=0}^{\infty} w_{v, m}^{(1)}\{\psi(\alpha v+m+\alpha)-\log (\beta)\} \\
& +\sum_{v, m=0}^{\infty} w_{v, m}^{(1)}(\alpha v+m+\alpha)+\frac{(a-1)}{c}\left\{\psi\left(\frac{a}{c}\right)-\psi\left(\frac{a}{c}+b\right)\right\}+(b-1)\left\{\psi(b)-\psi\left(\frac{a}{c}+b\right)\right\} .
\end{aligned}
$$

\subsection{Means Deviations}

The mean deviations of a random variable $X$ with respect to the mean and the median are

$$
\delta_{1}(X)=\int_{0}^{\infty}|x-\mu| f(x) \mathrm{d} x \text { and } \delta_{2}(X)=\int_{0}^{\infty}|x-M| f(x) \mathrm{d} x,
$$

respectively, where $\mu=\mathrm{E}(X)$ and $M=\operatorname{Median}(X)$ denotes the median. These quantities can be expressed as

$$
\delta_{1}(X)=2 \mu F(\mu)-2 \mu+2 T(\mu) \text { and } \delta_{2}(X)=2 T(M)-\mu,
$$

where $F(\mu)$ is the cdf of $X$ and $T(q)=\int_{q}^{\infty} x f(x) \mathrm{d} x$.

Based on the Corollary 1, the quantity $T(q)$ for the Mc- $\Gamma$ distribution becomes

$$
\begin{aligned}
T(q) & =\sum_{v, m=0}^{\infty} w_{v, m}^{(1)} \int_{q}^{\infty} x h(x ; \alpha v+m+\alpha, \beta) \mathrm{d} x \\
& =\sum_{v, m=0}^{\infty} w_{v, m}^{(1)}\left[1-\int_{0}^{q} x h(x ; \alpha v+m+\alpha, \beta) \mathrm{d} x\right] \\
& =\sum_{v, m=0}^{\infty} w_{v, m}^{(1)}\left[1-\frac{(\alpha v+m+v)}{\beta} \gamma_{1}(\alpha v+m+v+1, \beta q)\right]
\end{aligned}
$$

and $F(x)$ comes from equation (3).

\subsection{Bonferroni and Lorentz Curves}

Bonferroni $(B(\cdot))$ and Lorentz $(L(\cdot))$ curves have been applied in many fields such as economics, reliability, demography, insurance and medicine. Expressions of these measures for several important probability distributions were proposed by Giorgi \& Nadarajah (2010). For the Mc- $\Gamma$ distribution, these quantities are defined by

$$
B(p)=\frac{1}{p \mu} \int_{0}^{q} x f_{\mathrm{Mc}-\Gamma}(x) \mathrm{d} x \text { and } L(p)=\frac{1}{\mu} \int_{0}^{q} x f_{\mathrm{Mc}-\Gamma}(x) \mathrm{d} x=p B(p),
$$


where $q=F^{-1}(p)=Q_{\mathrm{Mc}-\Gamma}(p)$ is the Mc- $\Gamma$ quantile function for a given probability $p$ and $\mu=E(X)$. The Mc- $\Gamma$ quantile function can be calculated by inverting (5) and it will be studied in Section 3.8 (see also equation (8)). We obtain

$$
\begin{aligned}
B(p) & =\frac{1}{p \mu \beta} \sum_{v, m=0}^{\infty} w_{v, m}^{(1)}(\alpha v+m+\alpha) \int_{0}^{q} x h(x ; \alpha v+m+\alpha, \beta) \mathrm{d} x \\
& =\frac{1}{p}\left\{\frac{\sum_{v, m=0}^{\infty} w_{v, m}^{(1)}(\alpha v+m+\alpha) \gamma_{1}(\alpha v+m+\alpha, \beta q)}{\sum_{v, m=0}^{\infty} w_{v, m}^{(1)}(\alpha v+m+\alpha)}\right\} .
\end{aligned}
$$

\subsection{Skewness and Kurtosis}

We can express the Mc- $\Gamma$ quantile function in terms of the quantile functions of the $\Gamma(\alpha, \beta)$ and $\mathcal{B}\left(\frac{a}{c}, b\right)$ distributions, denoted by $Q_{\Gamma}(p)$ and $Q_{\mathcal{B}}(p)$, respectively. Using (3), we have $F_{\mathrm{Mc}-\Gamma}(x)=I_{\gamma_{1}(\alpha, \beta x)}\left(\frac{a}{c}, b\right)$. By inverting $I_{\gamma_{1}(\alpha, \beta x)^{c}}\left(\frac{a}{c}, b\right)=p$, we obtain $\gamma_{1}(\alpha, \beta x)^{c}=Q_{\mathcal{B}}(p)$, and the Mc- $\Gamma$ quantile function becomes

$$
Q_{\mathrm{Mc}-\Gamma}(p)=Q_{\Gamma}\left(Q_{\mathcal{B}}(p)^{1 / c}\right) .
$$

There are several robust measures in the literature for location and dispersion. The median, for example, can be used for location and the interquartile range. Both the median and the interquartile range are based on quantiles. From this fact, Bowley (1920) proposed a coefficient of skewness based on quantiles given by

$$
S K=\frac{Q(3 / 4)+Q(1 / 4)-2 Q(1 / 2)}{Q(3 / 4)-Q(1 / 4)},
$$

where $Q(\cdot)$ is the quantile function of a given distribution $F$. It can be shown that Bowley's coefficient of skewness takes the value zero for symmetric distributions. Additionally, its largest value is one and the lowest is -1 .

Moors (1986) demonstrated that the conventional measure of kurtosis may be interpreted as a dispersion around the values $\mu+\sigma$ and $\mu-\sigma$. Thus, the probability mass focuses around $\mu$ or on the tails of the distribution. Therefore, based on this interpretation, Moors (1988) proposed, as an alternative to the conventional coefficient of kurtosis, a robust measure based on octiles given by

$$
K R=\frac{(Q(7 / 8)-Q(5 / 8))+(Q(3 / 8)-Q(1 / 8))}{Q(6 / 8)-Q(2 / 8)} .
$$

Figs. 5(a)-5(b) provide the plots of the Bowley's skewness and Moors's kurtosis, respectively, for the proposed distribution.

\subsection{Order Statistics}

In the following discussion, we derive the order statistics and their $v$ th moments. The pdf of the $i$ th order statistic $X_{i: n}$, for $i=1,2, \ldots, n$, is given by

$$
f_{i: n}(x)=\frac{f(x)}{B(i, n-i+1)} \sum_{k=0}^{n-i}(-1)^{k}\left(\begin{array}{c}
n-i \\
k
\end{array}\right) F^{i+k-1}(x) .
$$

From equation (18) given in Appendix C, we have

$$
\left.f_{i: n} x\right)=\frac{1}{B(i, n-i+1)} \sum_{k=0}^{n-i}(-1)^{k}\left(\begin{array}{c}
n-i \\
k
\end{array}\right)\left\{\sum_{h_{1}, h_{2}, r, m, s_{1}, s_{2}=0}^{\infty} W_{h_{1}, h_{2}, r, m, s_{1}, s_{2}}^{(i+k,-1)}\right\} h\left(x ; \alpha s_{1}+s_{2}+\alpha+\alpha h_{2}+m, \beta\right) .
$$

Additionally, the $v$ th ordinary moment of $X_{i: n}$ is

$$
\mathrm{E}\left(X_{i: n}^{v}\right)=\int_{0}^{\infty} x^{v} f_{i: n}(x) \mathrm{d} x=\frac{1}{B(i, n-i+1)} \sum_{k=0}^{n-i}(-1)^{k}\left(\begin{array}{c}
n-i \\
k
\end{array}\right) \underbrace{\int_{0}^{\infty} x^{v} f(x) F^{i+k-1}(x) \mathrm{d} x}_{\mu_{v, i+k-1} \triangleq \mathrm{E}\left\{X^{v} F^{i+k-1}(X)\right\}},
$$

where the quantity $\mu_{v, i+k-1}$ is the probability weighted moment (PWM) of the Mc- $\Gamma$ distribution. From equation (9), we obtain

$$
\mathrm{E}\left(X_{i: n}^{v}\right)=\frac{1}{B(i, n-i+1)} \sum_{k=0}^{n-i}(-1)^{k}\left(\begin{array}{c}
n-i \\
k
\end{array}\right) \mu_{v, i+k-1},
$$


where

$$
\mu_{v, i+k-1}=\sum_{h_{1}, h_{2}, r, m, s_{1}, s_{2}=0}^{\infty} W_{h_{1}, h_{2}, r, m, s_{1}, s_{2}}^{(i+k-1)}\left[\frac{\Gamma\left(\alpha s_{1}+s_{2}+\alpha+\alpha h_{2}+m+v\right)}{\Gamma\left(\alpha s_{1}+s_{2}+\alpha+\alpha h_{2}+m\right) \beta^{v}}\right] .
$$

\subsection{Expansion for the Quantile Function}

Quantile functions are in widespread use in general statistics and often find representations in terms of lookup tables for key percentiles. An extensive discussion of the use of quantile functions in mainstream statistics is given in the book by Gilchrist (2000).

The quantile function $Q(u)$ usually does not have closed-form expressions for several important distributions, such as the normal, Student $t$, gamma and beta distributions. As a potential solution, this function can be expressed in terms of a power series of a transformed variable $v$, which takes the form $v=p(q u-t)^{\rho}$, for $p, q, t$ and $\rho$ known constants:

$$
Q(u)=\sum_{i=0}^{\infty} \epsilon_{i} v^{i}
$$

where the coefficients $\epsilon_{i}$ are suitably chosen real numbers. Steinbrecher (2002) explored the solution of this equation by standard power series methods.

According to Steinbrecher \& Shaw (2008), the following two results hold:

(r1): For the gamma distribution, equation (10) is defined by $v=\beta[\Gamma(\alpha+1) u]^{1 / \alpha}$ and

$$
\epsilon_{i}=m_{i}= \begin{cases}0, & \text { if } i=0 \\ 1, & \text { if } i=1 \\ a_{i+1}, & \text { if } i \geq 1\end{cases}
$$

where

$$
a_{i+1}=\frac{1}{i(\alpha+i)}\left\{\sum_{r=1}^{i} \sum_{s=1}^{i-s+1} a_{r} a_{s} a_{i-r-s+2} s(i-r-s+2)-\Delta(i) \sum_{r=2}^{i} a_{r} a_{i-r+2} r[r-\alpha-(1-\alpha)(i+2-r)]\right\},
$$

$\Delta(i)=0$ if $i<2$ and $\Delta(i)=1$ if $i \geq 2$. Here, the first coefficients are $a_{2}=1 /(\alpha+1), a_{3}=(3 \alpha+5) /\left[2(\alpha+1)^{2}(\alpha+2)\right], \ldots$ Hence, the power series for the gamma quantile is given by

$$
Q_{\Gamma}(u)=\sum_{i=0}^{\infty} m_{i}\left[\beta \Gamma(\alpha+1)^{1 / \alpha}\right]^{i} u^{i / \alpha} .
$$

(r2): For the beta quantile, the power series reduces to

$$
Q_{\mathcal{B}}(u)=\sum_{i=0}^{\infty} d_{i}^{\prime} u^{i c / a},
$$

where the transformed variable is $v=\left[a c^{-1} B\left(a c^{-1}, b\right) u\right]^{c a^{-1}}$,

$d_{i}^{\prime}=d_{i}\left[a c^{-1} B\left(a c^{-1}, b\right)\right]^{i c / a}$ and $d_{i}$ is given by

$$
\begin{gathered}
d_{i}= \begin{cases}0, & \text { if } i=0 \\
1, & \text { if } i=1 \\
\lambda_{i}, & \text { if } i \geq 2,\end{cases} \\
\lambda_{i}=\frac{1}{\left[i^{2}+(a / c-2) i+(1-a / c)\right]}\left\{\left(1-\delta_{i, 2}\right) \sum_{r=2}^{i-1} \lambda_{r} \lambda_{i+1-r}[r(1-a / c)(i-r)\right. \\
\left.-r(r-1)]+\sum_{r=1}^{i-1} \sum_{s=1}^{i-r} \lambda_{r} \lambda_{s} \lambda_{i+1-r-s}[r(r-a / c)+s(a / c+b-2)(i+1-r-s)]\right\},
\end{gathered}
$$


$\delta_{i, 2}=1$ if $i=2$ and $\delta_{i, 2}=0$ if $i \neq 2$. The first quantities are:

$$
\begin{aligned}
\lambda_{2}= & \frac{b-1}{a / c+1}, \\
\lambda_{3}= & \frac{(b-1)\left(a / c^{2}+3 b a / c-a / c+5 b-4\right)}{2(a / c+1)^{2}(a / c+2)}, \\
\lambda_{4}= & (b-1)\left[a / c^{4}+(6 b-1) a / c^{3}+(b+2)(8 b-5) a / c^{2}+\left(33 b^{2}-30 b+4\right) a / c\right. \\
& \quad+b(31 b-47)+18] /\left[3(a / c+1)^{3}(a / c+2)(a / c+3)\right], \ldots
\end{aligned}
$$

Inserting equation (11) into (8), we obtain

$$
Q_{\mathrm{Mc}-\Gamma}(p)=\sum_{i=0}^{\infty} m_{i}\left[\beta \Gamma(\alpha+1)^{1 / \alpha}\right]^{i}\left[Q_{\mathcal{B}}(p)\right]^{i / c \alpha} .
$$

Since $0<Q_{\mathcal{B}}(p)<1$ and $i / c \alpha>0$, we have

$$
\left[Q_{\mathcal{B}}(p)\right]^{i / c \alpha}=\sum_{k, v=0}^{\infty}(-1)^{k+v}\left(\begin{array}{c}
i / c \alpha \\
k
\end{array}\right)\left(\begin{array}{l}
k \\
v
\end{array}\right)\left[Q_{\mathcal{B}}(p)\right]^{v}
$$

Additionally, the beta quantile function (12) can be expressed as $p^{c / a} \sum_{j=0}^{\infty} w_{j} p^{j c / a}$, where $w_{j}=d_{j+1}^{\prime}=d_{j+1}\left[a c^{-1} B\left(a c^{-1}, b\right)\right]^{(j+1) c / a}$ for $j=0,1, \ldots$ In this case, the first two quantities are

$$
w_{0}=\left[a c^{-1} B\left(a c^{-1}, b\right)\right]^{c / a} \text { and } w_{1}=[(b-1) c /(a+c)]\left[a c^{-1} B\left(a c^{-1}, b\right)\right]^{2 c / a} .
$$

Applying the previous expressions in (13) and using the power series for the beta quantile function in (12), we obtain

$$
Q_{\mathrm{Mc}-\Gamma}(p)=\sum_{i, k, v=0}^{\infty} \underbrace{m_{i} \beta^{i} \Gamma(\alpha+1)^{i / \alpha}(-1)^{k+v}\left(\begin{array}{c}
(/ c \alpha \\
k
\end{array}\right)\left(\begin{array}{l}
k \\
v
\end{array}\right)}_{E_{i, k, v}}\left(p^{c / a} \sum_{j=0}^{\infty} w_{j} p^{j c / a}\right)^{v} .
$$

Thus, using a power series raised to a positive integer $v$ (Gradshteyn \& Ryzhik, 1980, p. 17), it follows that

$$
Q_{\mathrm{Mc}-\Gamma}(p)=\sum_{i, k, v, j=0}^{\infty} E_{i, k, v} s_{v, j} p^{c(v+j) a^{-1}}
$$

where $s_{v, 0}=w_{0}^{v}$ and $s_{v, j}=\left(j w_{0}\right)^{-1} \sum_{m=1}^{j}[m(v+1)-j] w_{m} s_{v, j-m}$. Notice that the coefficient $s_{v, j}$ can be recursively obtained from $\left\{s_{v, 0}, \ldots, s_{v, j-1}\right\}$ and $\left\{w_{0}, \ldots, w_{j}\right\}$.

Finally, setting $\ell=v+j$, the quantile function can be rewritten as

$$
Q_{\mathrm{Mc}-\Gamma}(p)=\sum_{\ell=0}^{\infty} N_{\ell} \pi^{\ell}
$$

where $N_{\ell}=\sum_{i, k=0}^{\infty} \sum_{v=0}^{\ell} E_{i, k, v} s_{v, \ell-v}$ and $\pi=p^{c a^{-1}}$. The last expansion can be used as an alternative way for calculating some mathematical quantities of the new distribution. For example, the result

$$
\mathrm{E}\left(X^{n}\right)=\int_{0}^{\infty} x^{n} f(x) \mathrm{d} x=\int_{0}^{1} Q(p)^{n} \mathrm{~d} p
$$

combined with a power series raised to a positive integer, leads to an alternative form for the ordinary moments

$$
\mathrm{E}\left(X^{n}\right)=a c^{-1} \int_{0}^{1}\left(\sum_{\ell=0}^{\infty} N_{\ell} \pi^{\ell}\right)^{n} \pi^{a c^{-1}-1} \mathrm{~d} \pi=a c^{-1} \sum_{\ell=0}^{\infty} N_{n, \ell}^{\prime} \int_{0}^{1} \pi^{\ell+a c^{-1}-1} \mathrm{~d} \pi=a c^{-1} \sum_{\ell=0}^{\infty} \frac{N_{n, \ell}^{\prime}}{\ell+a c^{-1}},
$$

where $N_{n, 0}^{\prime}=N_{0}^{n}$ and $N_{n, \ell}^{\prime}=\left(\ell N_{0}\right)^{-1} \sum_{m=1}^{\ell}[m(n+1)-\ell] N_{m} N_{m, \ell-m}^{\prime}$. 


\section{Estimation}

The parameters of the Mc- $\Gamma$ distribution can be estimated by the method of maximum likelihood. Let $x_{1}, \cdots, x_{n}$ be a random sample of size $n$ from the $\operatorname{Mc}-\Gamma(\alpha, \beta, a, b, c)$ distribution given by (4). The log-likelihood function for the vector of parameters $\theta=(\alpha, \beta, a, b, c)^{\top}$ can be expressed as

$$
\begin{aligned}
l(\theta)= & n \log c+n \alpha \log \beta-n \log B\left(\frac{a}{c}, b\right)-n \log \Gamma(\alpha)+(\alpha-1) \sum_{i=1}^{n} \log x_{i}-\beta \sum_{i=1}^{n} x_{i}+(a-1) \sum_{i=1}^{n} \log \gamma_{1}\left(\alpha, \beta x_{i}\right) \\
& +(b-1) \sum_{i=1}^{n} \log \left[1-\gamma_{1}\left(\alpha, \beta x_{i}\right)^{c}\right] .
\end{aligned}
$$

The components of the score vector $\mathbf{U}(\theta)$ are

$$
\begin{aligned}
U_{\alpha}(\theta)=\frac{\partial l(\theta)}{\partial \alpha}=n \log \beta-n \psi(\alpha)+\sum_{i=1}^{n} \log \left(x_{i}\right)+(a-1) \sum_{i=1}^{n}\left\{\frac{\partial \gamma_{1}\left(\alpha, \beta x_{i}\right) / \partial \alpha}{\gamma_{1}\left(\alpha, \beta x_{i}\right)}\right\}-c(b-1) \sum_{i=1}^{n}\left\{\frac{\gamma_{1}\left(\alpha, \beta x_{i}\right)^{c-1} \partial \gamma_{1}\left(\alpha, \beta x_{i}\right) / \partial \alpha}{1-\gamma_{1}\left(\alpha, \beta x_{i}\right)^{c}}\right\} \\
U_{\beta}(\theta)=\frac{\partial l(\theta)}{\partial \beta}=\frac{n \alpha}{\beta}-\sum_{i=1}^{n} x_{i}+(a-1) \sum_{i=1}^{n} x_{i}\left\{\frac{\partial \gamma_{1}\left(\alpha, \beta x_{i}\right) / \partial \beta}{\gamma_{1}\left(\alpha, \beta x_{i}\right)}\right\}-c(b-1) \sum_{i=1}^{n} x_{i}\left\{\frac{\gamma_{1}\left(\alpha, \beta x_{i}\right)^{c-1} \partial \gamma_{1}\left(\alpha, \beta x_{i}\right) / \partial \beta}{1-\gamma_{1}\left(\alpha, \beta x_{i}\right)^{c}}\right\} \\
U_{a}(\theta)=\frac{\partial l(\theta)}{\partial a}=\frac{n}{c}\left[\psi\left(\frac{a}{c}+b\right)-\psi\left(\frac{a}{c}\right)\right]+\sum_{i=1}^{n} \log \left[\gamma_{1}\left(\alpha, \beta x_{i}\right)\right] \\
U_{b}(\theta)=\frac{\partial l(\theta)}{\partial b}=n\left[\psi\left(\frac{a}{c}+b\right)-\psi(b)\right]+\sum_{i=1}^{n} \log \left[1-\gamma_{1}\left(\alpha, \beta x_{i}\right)^{c}\right] \\
U_{c}(\theta)=\frac{\partial l(\theta)}{\partial c}=\frac{n}{c}-\frac{n a}{c^{2}}\left[\psi\left(\frac{a}{c}+b\right)-\psi\left(\frac{a}{c}\right)\right]-(b-1) \sum_{i=1}^{n}\left\{\frac{\gamma_{1}\left(\alpha, \beta x_{i}\right)^{c} \log \gamma_{1}\left(\alpha, \beta x_{i}\right)}{1-\gamma_{1}\left(\alpha, \beta x_{i}\right)^{c}}\right\}
\end{aligned}
$$

These expressions depend on the quantities $\partial \gamma_{1}(\cdot) / \partial \alpha$ and $\partial \gamma_{1}(\cdot) / \partial \beta$. Now, we provide formulas for these quantities. Using MATHEMATICA, we obtain

$$
\frac{\partial \gamma(\alpha, \beta x)}{\partial \alpha}=\Gamma(\alpha) \psi(\alpha)-\log (\beta x) \gamma(\alpha, \beta x)-G_{23}^{30}\left(\left.\beta x\right|_{0,0, \alpha} ^{1,1}\right),
$$

where $G_{23}^{30}(\cdot)$ is a particular case of the Meijer G-function (note 2) given by

$$
G_{23}^{30}\left(\left.\beta x\right|_{0,0, \alpha} ^{1,1}\right)=\frac{(\beta x)^{\alpha}{ }_{2} F_{2}(\{\alpha, \alpha\} ;\{\alpha+1, \alpha+1\} ;-\beta x)}{\alpha^{2}}-\Gamma(\alpha) \log (\beta x)+\Gamma(\alpha) \psi(\alpha),
$$

and ${ }_{2} F_{2}(\cdot ; \cdot ; \cdot)$ denotes the hypergeometric function defined by

$$
{ }_{2} F_{2}\left(\left\{a_{1}, a_{2}\right\} ;\left\{b_{1}, b_{2}\right\} ; z\right)=\sum_{j=0}^{\infty} \frac{\left(a_{1}\right)_{j}\left(a_{2}\right)_{j}}{\left(b_{1}\right)_{j}\left(b_{2}\right)_{j}} \frac{z^{j}}{j !},
$$

where, for some parameter $\mu$, the Pochhammer symbol $(\mu)_{j}$ is defined by

$$
(\mu)_{0}=1, \quad(\mu)_{j}=\mu(\mu+1) \cdots(\mu+j-1), \quad j=1,2, \ldots
$$

We obtain

$$
\frac{\partial \gamma(\alpha, \beta x)}{\partial \alpha}=\log (\beta x)\{\Gamma(\alpha)-\gamma(\alpha, \beta x)\}-\frac{(\beta x)^{\alpha}{ }_{2} F_{2}(\{\alpha, \alpha\} ;\{\alpha+1, \alpha+1\} ;-\beta x)}{\alpha^{2}} .
$$

Finally, we have

$$
\frac{\partial \gamma_{1}(\alpha, \beta x)}{\partial \alpha}=\log (\beta x)\left\{1-\gamma_{1}(\alpha, \beta x)\right\}-\frac{(\beta x)^{\alpha}{ }_{2} F_{2}(\{\alpha, \alpha\} ;\{\alpha+1, \alpha+1\} ;-\beta x)}{\Gamma(\alpha) \alpha^{2}}-\psi(\alpha) \gamma_{1}(\alpha, \beta x) .
$$

Additionally,

$$
\frac{\partial \gamma_{1}(\alpha, \beta x)}{\partial \beta}=\frac{x^{\alpha} \beta^{\alpha-1} \exp \{-\beta x\}}{\Gamma(\alpha)} .
$$


The maximum likelihood estimate (MLE) $\hat{\theta}$ of $\theta$ is the solution of the system of nonlinear equations $\mathbf{U}(\theta)=\mathbf{0}$. For interval estimation and tests of hypotheses on the parameters in $\theta$, we require the $5 \times 5$ unit observed information matrix $J=J(\theta)$, whose elements are given in Appendix D. Under certain regularity conditions for the likelihood function, confidence intervals and hypothesis tests can be constructed using the fact that the asymptotic distribution of the MLE $\hat{\theta}$ is $\sqrt{n}(\hat{\theta}-\theta) \sim N_{p}\left(0, I(\theta)^{-1}\right)$, where $I(\theta)$ is the Fisher information matrix and $p$ is the number of model parameters (Sen \& Singer, 1993). We can substitute $I(\theta)$ by $J(\hat{\theta})$, i.e., the observed information matrix evaluated at $\hat{\theta}$.

The multivariate normal $N_{5}\left(0, J(\hat{\theta})^{-1}\right)$ approximated distribution can be used to obtain confidence intervals for the individual parameters. We can compute the maximum values of the unrestricted and restricted log-likelihoods to define likelihood ratio (LR) statistics for testing some sub-models of the Mc- $\Gamma$ distribution. We may be interested to check if the fit using the Mc- $\Gamma$ distribution is statistically "superior" to a fit using the $\mathcal{B}-\Gamma, \mathrm{Kw}-\Gamma$ and $\Gamma$ distributions for a given data set.

\section{Application}

In this section, we present and compare the performance of the Mc- $\Gamma$ distribution and its $\mathrm{Kw}-\Gamma, \mathcal{B}-\Gamma$ and $\Gamma$ sub-models to describe a real data set from USS Halfbeak diesel engine. The data were previously studied by (Ascher, 1984, p. 75) and (Meeker, 1998, p. 415). They represent times of unscheduled maintenance actions for the USS Halfbeak number 4 main propulsion diesel engine over 25.518 operating hours. Table 1 gives some statistical measures for these data. These values indicate that the empirical distribution is skewed to the left and platycurtic.

In order to compare the fits of the Mc- $\Gamma, \mathcal{B}-\Gamma, \mathrm{Kw}-\Gamma$ and $\Gamma$ distributions, the maximum likelihood method was adopted using the subroutine 'NLMixed' in the SAS software. Table 2 lists the MLEs, their standard errors, and three goodnessof-fit statistics: AIC (Akaike Information Criterion), BIC (Bayesian Information Criterion) and CAIC (Consistent Akaike Information Criterion). The lowest values of these statistics correspond to the Mc- $\Gamma$ distribution. Fig. 6 shows that the Mc- $\Gamma$ distribution provides a closer fit to the histogram of the data than the other three sub-models.

In order to verify the importance of the Mc- $\Gamma$ distribution in relation to its sub-models by means of hypothesis tests, we consider the LR statistic given by

$$
\Lambda=2\{l(\widehat{\theta})-l(\widetilde{\theta})\}=2\{l(\widehat{\alpha}, \widehat{\beta}, \widehat{a}, \widehat{b}, \widehat{c})-l(\widetilde{\alpha}, \widetilde{\beta}, \widetilde{a}, \widetilde{b}, \widetilde{c})\}
$$

where $\widehat{\theta}$ and $\widetilde{\theta}$ are the MLEs of the parameter $\theta$ under the alternative and null hypotheses, respectively. The LR statistic can be used to verify if the fit of the Mc- $\Gamma$ distribution outperforms statistically those fits of their sub-models. Table 3 lists the values of the LR statistics in order to quantify the adequacy of the new distribution. The results provide evidence that the additional parameters of the new distribution are statistically significant in these comparisons, justifying its use for modelling positive real data sets.

\section{Conclusions}

We present a new five-parameter distribution, called the McDonald gamma (Mc-Г) distribution, which includes as special cases several commonly used distributions in the literature. Further, the new distribution has proved to be versatile and analytically tractable. We provide a mathematical treatment of this distribution including analytical expressions for the moments, moment generating function, log-moment, mean deviations, Lorentz and Bonferroni curves, order statistics, entropy and quantile function. Additionally, maximum likelihood estimation of the model parameters was discussed and the observed information matrix was derived.

An application to real data was performed in order to quantifying the adequacy of the Mc- $\Gamma$ distribution and some of its sub-models. The results indicate that the proposed distribution outperforms its main sub-models.

\section{Acknowledgment}

The authors are very grateful to a referee and an associate editor for helpful comments that considerably improved the paper. We gratefully acknowledge financial support from CNPq and CAPES.

\section{Appendix}

A. Proof of Theorem 1 and Corollary 1: representation in power series for $f_{M c-\Gamma}(x)^{s}$, where s is a positive real number.

First, consider the derivation of the following expression:

$$
\delta(\alpha, \beta, a, b, c, s)=\left\{\gamma_{1}(\alpha, \beta x)^{a-1}\left[1-\gamma_{1}(\alpha, \beta x)^{c}\right]^{b-1}\right\}^{s} .
$$


Since $\gamma_{1}(\alpha, \beta x)<1$ and $s(a-1), c>0$, we have

$$
\left[1-\gamma_{1}(\alpha, \beta x)^{c}\right]^{s(b-1)}=\sum_{i=0}^{\infty}(-1)^{i}\left[\gamma_{1}(\alpha, \beta x)\right]^{c i(}\left(\begin{array}{c}
s(b-1) \\
i
\end{array}\right) .
$$

Thus,

$$
\delta(\alpha, \beta, a, b, c, s)=\sum_{i=0}^{\infty}(-1)^{i}\left[\gamma_{1}(\alpha, \beta x)\right]^{i+s(a-1)\left(\begin{array}{c}
s(b-1) \\
i
\end{array}\right) .}
$$

The kernel of the above function can be represented as

$$
\begin{aligned}
{\left[\gamma_{1}(\alpha, \beta x)\right]^{c i+s(a-1)} } & =\left\{1-\left[1-\gamma_{1}(\alpha, \beta x)\right]\right\}^{c i+s(a-1)} \\
& =\sum_{k=0}^{\infty}(-1)^{k}\left[1-\gamma_{1}(\alpha, \beta x)\right]^{k}\left(\begin{array}{c}
c i+s(a-1) \\
k
\end{array}\right) \\
& =\sum_{k=0}^{\infty}(-1)^{k}\left(\begin{array}{c}
c i+s(a-1) \\
k
\end{array}\right) \sum_{v=0}^{\infty}(-1)^{v} \gamma_{1}(\alpha, \beta x)^{v}\left(\begin{array}{l}
k \\
v
\end{array}\right) .
\end{aligned}
$$

Combining this result with the power series (7), we obtain

$$
\delta(\alpha, \beta, a, b, c, s)=\sum_{i, k, v=0}^{\infty}(-1)^{i+k+v}\left(\begin{array}{c}
k \\
v
\end{array}\right)\left(\begin{array}{c}
s(b-1) \\
i
\end{array}\right)\left(\begin{array}{c}
c i+s(a-1) \\
k
\end{array}\right)\left\{\frac{(\beta x)^{\alpha}}{\Gamma(\alpha)}\right\}^{v}\left\{\sum_{m=0}^{\infty} \frac{(-\beta)^{m} x^{m}}{(\alpha+m) m !}\right\}^{v} .
$$

We consider the result concerning a power series raised to a positive integer $v$ (Gradshteyn \& Ryzhik, 1980, p. 17) given by

$$
\{\sum_{m=0}^{\infty} \underbrace{\frac{(-\beta)^{m}}{(\alpha+m) m !}}_{a_{m}} x^{m}\}^{v}=\sum_{m=0}^{\infty} t_{m, v} x^{m},
$$

where $t_{0, v}=\alpha^{-v}$ and $t_{m, v}=m^{-1} \alpha \sum_{h=1}^{m}[h(v+1)-m] a_{h} t_{m-h, v}$ for $m \geq 1$. Hence,

$$
\delta(\alpha, \beta, a, b, c, s)=\sum_{i, k, v, m=0}^{\infty}(-1)^{i+k+v}\left(\begin{array}{c}
k \\
v
\end{array}\right)\left(\begin{array}{c}
s(b-1) \\
i
\end{array}\right)\left(\begin{array}{c}
c i+s(a-1) \\
k
\end{array}\right)\left\{\frac{\beta^{v \alpha} x^{\alpha v+m} t_{m, v}}{\Gamma(\alpha)^{v}}\right\} .
$$

Now, we provide a power series for $f_{\mathrm{Mc}-\Gamma}(x)^{s}$. From equation (15), this quantity can be rewritten as

$$
\begin{aligned}
& f_{\mathrm{Mc}-\Gamma}(x ; \alpha, \beta, a, b, c,)^{s}=\left\{\frac{c \beta^{\alpha} x^{\alpha-1} \exp (-\beta x)}{\Gamma(\alpha) B\left(\frac{a}{c}, b\right)}\right\}^{s} \delta(\alpha, \beta, a, b, c, s) \\
= & \sum_{v, m=0}^{\infty} \underbrace{\left.\left\{\frac{\Gamma(\alpha v+m+1+s(\alpha-1))}{\beta^{m+1-s} \Gamma(\alpha)^{v}} t_{m, v}\right\} \sum_{i, k=0}^{\infty}(-1)^{i+k+v}\left(\begin{array}{c}
k \\
v
\end{array}\right)\left(\begin{array}{c}
s(b-1) \\
i
\end{array}\right)\left(\begin{array}{c}
(i+s(a-1) \\
k
\end{array}\right)\right\}}_{w_{v, m}^{(s)}} \times \underbrace{\frac{\beta^{\alpha v+m+1+s(\alpha-1)} x^{\alpha v+m+1+s(\alpha-1)-1} \exp (-\beta x)}{\Gamma(\alpha v+m+1+s(\alpha-1))}}_{h(x ; \alpha v+m+1+s(\alpha-1), \beta)} \\
= & \sum_{v, m=0}^{\infty} w_{v, m}^{(s)} h(x ; \alpha v+m+1+s(\alpha-1), \beta),
\end{aligned}
$$

where $h(x ; \cdot, \cdot)$ denotes the gamma density function. From this result, we have

$$
f_{\mathrm{Mc}-\Gamma}(x ; \alpha, \beta, a, b, c,)=\sum_{v, m=0}^{\infty} w_{v, m}^{(1)} h(x ; \alpha v+m+\alpha, \beta),
$$

i.e., the density function of the Mc- $\Gamma$ distribution is a double linear combination of gamma density functions.

B. Representation for $f_{M c-\Gamma}(x)$ and $F_{M c-\Gamma}(x)$ as functions of the exponentiated gamma distribution.

Applying the result (14) for $s=1$ in (4), this density can be rewritten as

$$
f_{\mathrm{Mc}-\Gamma}(x ; \alpha, \beta, a, b, c)=\sum_{k=0}^{\infty}\left[(-1)^{k}\left(\begin{array}{c}
b-1 \\
k
\end{array}\right)\right]\left[\frac{c \beta}{\beta\left(\frac{a}{c}, b\right)}\right] \frac{(\beta x)^{\alpha-1} \exp (-\beta x)}{\Gamma(\alpha)}\left[\gamma_{1}(\alpha, \beta x)\right]^{c k+a-1} .
$$


Setting $u=\beta x$, we have

$$
\begin{aligned}
f_{\mathrm{Mc}-\Gamma} & (u ; \alpha, \beta, a, b, c)= \\
& =\sum_{k=1}^{\infty}\left[(-1)^{k}\left(\begin{array}{c}
b-1 \\
k
\end{array}\right)\right]\left[\frac{c \beta}{\beta\left(\frac{a}{c}, b\right)}\right] \frac{u^{\alpha-1} \exp (-u)}{\Gamma(\alpha)}\left[\gamma_{1}(\alpha, u)\right]^{c k+a-1} \\
& =\sum_{k=0}^{\infty}\left[(-1)^{k}\left(\begin{array}{c}
b-1 \\
k
\end{array}\right)\right]\left[\frac{c \beta}{(c k+a) \beta\left(\frac{a}{c}, b\right)}\right] \underbrace{(c k+a) \frac{u^{\alpha-1} \exp (-u)}{\Gamma(\alpha)}\left[\gamma_{1}(\alpha, u)\right]^{c k+a-1}}_{h_{\mathrm{EG}}(u ; \alpha, c k+a)} \\
& =\sum_{k=0}^{\infty} \underbrace{\left[(-1)^{k}\left(\begin{array}{c}
b-1 \\
k
\end{array}\right)\right]\left[\frac{c \beta}{(c k+a) B\left(\frac{a}{c}, b\right)}\right]}_{w_{k}^{\prime}} h_{\mathrm{EG}}(u ; \alpha, c k+a),
\end{aligned}
$$

where $h_{\mathrm{EG}}\left(x ; k_{1}, k_{2}\right)$ is given by

$$
h_{\mathrm{EG}}\left(x ; k_{1}, k_{2}\right)=\frac{k_{2} x^{k_{1}-1} \exp (-x)}{\Gamma\left(k_{1}\right)}\left\{\frac{\gamma\left(k_{1}, x\right)}{\Gamma\left(k_{1}\right)}\right\}^{k_{2}-1},
$$

which is the exponentiated standard gamma density with parameters $k_{1}, k_{2}>0$ (termed here by $\mathrm{EG}\left(k_{1}, k_{2}\right)$ ) (Nadarajah \& Kotz, 2006). In this case, its cdf can be expressed as

$$
F_{\mathrm{Mc}-\Gamma}(u ; \alpha, \beta, a, b, c)=\sum_{k=0}^{\infty} w_{k}^{\prime} H_{\mathrm{EG}}(u ; \alpha, c k+a)=\sum_{k=0}^{\infty} w_{k}^{\prime}\left[\gamma_{1}(\alpha, \beta u)\right]^{c k+a} .
$$

C. A linear combination for the quantity $f_{M c-\Gamma}(x) F_{M c-\Gamma}(x)^{v 1}$, where $v_{1}$ is a positive integer number.

From equations (16) and (17), we can write

$$
f_{M c-\Gamma}(x) F_{M c-\Gamma}(x)^{v_{1}}=\left[\gamma_{1}^{a}(\alpha, \beta u)\right]^{\nu_{1}}\left[\sum_{s_{1}, s_{2}=0}^{\infty} w_{s_{1}, s_{2}}^{(1)} h\left(x ; \alpha s_{1}+s_{2}+\alpha, \beta\right)\right]\left\{\sum_{k=0}^{\infty} w_{k}^{\prime}\left[\gamma_{1}(\alpha, \beta u)\right]^{c k}\right\}^{v_{1}} .
$$

Since $v_{1}$ is a positive integer number, we obtain

$$
f_{\mathrm{Mc}-\Gamma}(x) F_{\mathrm{Mc}-\Gamma}(x)^{v_{1}}=\left[\sum_{s_{1}, s_{2}=0}^{\infty} w_{s_{1}, s_{2}}^{(1)} h\left(x ; \alpha s_{1}+s_{2}+\alpha, \beta\right)\right]\left\{\sum_{k=0}^{\infty} s_{k, v_{1}}\left[\gamma_{1}(\alpha, \beta u)\right]^{c k+a v_{1}}\right\},
$$

where $s_{0, v_{1}}=w_{0}^{\prime v_{1}}$ and $s_{k, v_{1}}=\left(w_{0}^{\prime v_{1}} k\right)^{-1} \sum_{i=1}^{k}\left(i v_{1}-k-i\right) w_{i}^{\prime} s_{k-i, v_{1}}$ for $k \geq 1$.

Now, following similar arguments of the result (15), we have

$$
\left[\gamma_{1}(\alpha, \beta x)\right]^{c k+a v_{1}}=\sum_{h_{1}, h_{2}, m=0}^{\infty}(-1)^{h_{1}+h_{2}}\left(\begin{array}{c}
h_{1} \\
h_{2}
\end{array}\right)\left(\begin{array}{c}
c k+a v_{1} \\
h_{1}
\end{array}\right)\left\{\frac{t_{m, h_{2}} x^{\alpha h_{2}+m} \beta^{\alpha h_{2}}}{\Gamma(\alpha)^{h_{2}}}\right\} .
$$

Thus,

$$
\begin{aligned}
& f_{\mathrm{Mc}-\Gamma}(x) F_{\mathrm{Mc}-\Gamma}(x)^{v_{1}}= \\
& =\sum_{h_{1}, h_{2}, k, m, s_{1}, s_{2}=0}^{\infty} s_{k, v_{1}} w_{s_{1}, s_{2}}^{(1)}(-1)^{h_{1}+h_{2}}\left(\begin{array}{c}
h_{1} \\
h_{2}
\end{array}\right)\left(\begin{array}{c}
c k+a v_{1} \\
h_{1}
\end{array}\right)\left\{\frac{t_{m, h_{2}} \beta^{\alpha h_{2}}}{\Gamma(\alpha)^{h_{2}}}\right\} \underbrace{x^{\alpha h_{2}+m} h\left(x ; \alpha s_{1}+s_{1}+s_{2}+\alpha+\alpha h_{2}+m, \beta\right)}_{\left.\frac{\Gamma\left(s s_{1}+s_{2}+\alpha+\alpha h_{2}+m\right)}{\beta^{\alpha h_{2}+m} \Gamma\left(\alpha x s_{1}+s_{2}+\alpha\right)}\right)} \\
& =\sum_{h_{1}, h_{2}, k, m, s_{1}, s_{2}=0}^{\infty} W_{h_{1}, h_{2}, k, m, s_{1}, s_{2}}^{\left(v_{1}\right)} h\left(x ; \alpha s_{1}+s_{2}+\alpha+\alpha h_{2}+m, \beta\right),
\end{aligned}
$$

where

$$
W_{h_{1}, h_{2}, k, m, s_{1}, s_{2}}^{\left(v_{1}\right)}=s_{k, v_{1}} w_{s_{1}, s_{2}}^{(1)}(-1)^{h_{1}+h_{2}}\left(\begin{array}{c}
h_{1} \\
h_{2}
\end{array}\right)\left(\begin{array}{c}
c k+a v_{1} \\
h_{1}
\end{array}\right)\left\{\frac{t_{m, h_{2}} \Gamma\left(\alpha s_{1}+s_{2}+\alpha+\alpha h_{2}+m\right)}{\beta^{m} \Gamma\left(\alpha s_{1}+s_{2}+\alpha\right) \Gamma(\alpha)^{h_{2}}}\right\} .
$$




\section{Information Matrix.}

The elements of the observed information matrix $J(\theta)$ for the parameters $(\alpha, \beta, a, b, c)$ are:

$J_{\alpha \alpha}=-n \psi^{\prime}(\alpha)+(a-1) \sum_{i=1}^{n} y_{\alpha \alpha 1}\left(x_{i}\right)-c(b-1) \sum_{i=1}^{n} y_{\alpha \alpha 2}\left(x_{i}\right)$,

$J_{\alpha \beta}=\frac{n}{\beta}+(a-1) \sum_{i=1}^{n} x_{i} y_{\alpha \beta 1}\left(x_{i}\right)-c(b-1) \sum_{i=1}^{n} x_{i} y_{\alpha \beta 2}\left(x_{i}\right)$,

$J_{\alpha a}=\sum_{i=1}^{n} y_{\alpha a}\left(x_{i}\right)$,

$J_{\alpha b}=-c \sum_{i=1}^{n} y_{\alpha b}\left(x_{i}\right)$

$J_{\alpha c}=-(b-1)\left[\sum_{i=1}^{n} y_{\alpha b}\left(x_{i}\right)+c \sum_{i=1}^{n} y_{\alpha c}\left(x_{i}\right)\right], J_{\beta \beta}=$

$J_{\beta a}=\sum_{i=1}^{n} x_{i} y_{\beta a}\left(x_{i}\right)$,

$J_{\beta b}=-c \sum_{i=1}^{n} x_{i} y_{\beta b}\left(x_{i}\right)$

$J_{\beta c}=-(b-1)\left[\sum_{i=1}^{n} x_{i} y_{\beta b}\left(x_{i}\right)+c \sum_{i=1}^{n} x_{i} y_{\beta c 2}\left(x_{i}\right)\right]$,

$J_{a a}=\frac{n}{c^{2}}\left[\psi^{\prime}\left(\frac{a}{c}+b\right)-\psi^{\prime}\left(\frac{a}{c}\right)\right]$,

$J_{a b}=\frac{n}{c} \psi^{\prime}\left(\frac{a}{c}+b\right)$,

$J_{a c}=\frac{n}{c^{2}}\left[\psi\left(\frac{a}{c}\right)-\psi\left(\frac{a}{c}+b\right)\right]+\frac{n a}{c^{3}}\left[\psi^{\prime}\left(\frac{a}{c}\right)-\psi^{\prime}\left(\frac{a}{c}+b\right)\right]$,

$J_{b b}=n\left[\psi^{\prime}\left(\frac{a}{c}+b\right)-\psi^{\prime}(b)\right]$,

$J_{b c}=-\frac{n a}{c^{2}} \psi^{\prime}\left(\frac{a}{c}+b\right)-\sum_{i=1}^{n} y_{b c}\left(x_{i}\right)$,

$J_{c c}=-\frac{n}{c^{2}}+\frac{2 n a}{c^{3}}\left[\psi\left(\frac{a}{c}+b\right)-\psi\left(\frac{a}{c}\right)\right]+\frac{n a^{2}}{c^{4}}\left[\psi^{\prime}\left(\frac{a}{c}+b\right)-\psi^{\prime}\left(\frac{a}{c}\right)\right]-(b-1) \sum_{i=1}^{n} y_{c c}\left(x_{i}\right)$,

where the quantities $y_{\alpha \alpha 1}\left(x_{i}\right), y_{\alpha \alpha 2}\left(x_{i}\right), y_{\alpha \beta 1}\left(x_{i}\right), y_{\alpha \beta 2}\left(x_{i}\right), y_{\alpha a}\left(x_{i}\right), y_{\alpha b}\left(x_{i}\right), y_{\alpha c}\left(x_{i}\right), y_{\beta \beta 1}\left(x_{i}\right), y_{\beta \beta 2}\left(x_{i}\right), y_{\beta a}\left(x_{i}\right), y_{\beta b}\left(x_{i}\right), y_{\beta c}\left(x_{i}\right)$, $y_{b c}\left(x_{i}\right)$ and $y_{c c}\left(x_{i}\right)$ are given in Appendix E.

E. Auxiliary terms for the observed information matrix.

$$
\begin{aligned}
& y_{\alpha \alpha 1}\left(x_{i}\right)=\frac{\gamma_{1}\left(\alpha, \beta x_{i}\right)\left\{\partial^{2}\left[\gamma_{1}\left(\alpha, \beta x_{i}\right)\right] / \partial \alpha^{2}\right\}-\left\{\partial\left[\gamma_{1}\left(\alpha, \beta x_{i}\right)\right] / \partial \alpha\right\}^{2}}{\gamma_{1}\left(\alpha, \beta x_{i}\right)^{2}}, \\
& y_{\alpha \alpha 2}\left(x_{i}\right)=\left\{\frac{\partial\left[\gamma_{1}\left(\alpha, \beta x_{i}\right)\right]}{\partial \alpha}\right\}^{2} \frac{\left[\gamma_{1}\left(\alpha, \beta x_{i}\right)^{c-2}(c-1)+\gamma_{1}\left(\alpha, \beta x_{i}\right)^{2(c-1)}\right]}{\left[1-\gamma_{1}\left(\alpha, \beta x_{i}\right)^{c}\right]^{2}}+\frac{\partial^{2}\left[\gamma_{1}\left(\alpha, \beta x_{i}\right)\right]}{\partial \alpha^{2}} \frac{\left[\gamma_{1}\left(\alpha, \beta x_{i}\right)^{c-1}-\gamma_{1}\left(\alpha, \beta x_{i}\right)^{2 c-1}\right]}{\left[1-\gamma_{1}\left(\alpha, \beta x_{i}\right)^{c}\right]^{2}}, \\
& y_{\alpha \beta 1}\left(x_{i}\right)=\frac{\gamma_{1}\left(\alpha, \beta x_{i}\right)\left\{\partial^{2}\left[\gamma_{1}\left(\alpha, \beta x_{i}\right)\right] / \partial \alpha \partial \beta\right\}-\left\{\partial\left[\gamma_{1}\left(\alpha, \beta x_{i}\right)\right] / \partial \alpha\right\}\left\{\partial\left[\gamma_{1}\left(\alpha, \beta x_{i}\right)\right] / \partial \beta\right\}}{\gamma_{1}\left(\alpha, \beta x_{i}\right)^{2}}, \\
& y_{\alpha \beta 2}\left(x_{i}\right)=\frac{\partial\left[\gamma_{1}\left(\alpha, \beta x_{i}\right)\right]}{\partial \alpha} \frac{\partial\left[\gamma_{1}\left(\alpha, \beta x_{i}\right)\right]}{\partial \beta} \frac{\left[\gamma_{1}\left(\alpha, \beta x_{i}\right)^{c-2}(c-1)+\gamma_{1}\left(\alpha, \beta x_{i}\right)^{2(c-1)}\right]}{\left[1-\gamma_{1}\left(\alpha, \beta x_{i}\right)^{c}\right]^{2}}+\frac{\partial^{2}\left[\gamma_{1}\left(\alpha, \beta x_{i}\right)\right]}{\partial \alpha \partial \beta} \frac{\left[\gamma_{1}\left(\alpha, \beta x_{i}\right)^{c-1}-\gamma_{1}\left(\alpha, \beta x_{i}\right)^{2 c-1}\right]}{\left[1-\gamma_{1}\left(\alpha, \beta x_{i}\right)^{c}\right]^{2}},
\end{aligned}
$$




$$
\begin{aligned}
y_{\alpha a}\left(x_{i}\right)= & \frac{\partial\left[\gamma_{1}\left(\alpha, \beta x_{i}\right)\right] / \partial \alpha}{\gamma_{1}\left(\alpha, \beta x_{i}\right)}, \\
y_{\alpha b}\left(x_{i}\right) & =\frac{\gamma_{1}\left(\alpha, \beta x_{i}\right)^{c-1}\left\{\partial\left[\gamma_{1}\left(\alpha, \beta x_{i}\right)\right] / \partial \alpha\right\}}{1-\gamma_{1}\left(\alpha, \beta x_{i}\right)^{c}}, \\
y_{\alpha c}\left(x_{i}\right) & =\frac{\gamma_{1}\left(\alpha, \beta x_{i}\right)^{c-1} \log \left[\gamma_{1}\left(\alpha, \beta x_{i}\right)\right]\left\{\partial\left[\gamma_{1}\left(\alpha, \beta x_{i}\right)\right] / \partial \alpha\right\}}{\left[1-\gamma_{1}\left(\alpha, \beta x_{i}\right)^{c}\right]^{2}}, \\
y_{\beta \beta 1}\left(x_{i}\right) & =\frac{\gamma_{1}\left(\alpha, \beta x_{i}\right)\left\{\partial^{2}\left[\gamma_{1}\left(\alpha, \beta x_{i}\right)\right] / \partial \beta^{2}\right\}-\left\{\partial\left[\gamma_{1}\left(\alpha, \beta x_{i}\right)\right] / \partial \beta\right\}^{2}}{\gamma_{1}\left(\alpha, \beta x_{i}\right)^{2}}, \\
y_{\beta \beta 2}\left(x_{i}\right) & =\left\{\frac{\partial\left[\gamma_{1}\left(\alpha, \beta x_{i}\right)\right]}{\partial \beta}\right\}^{2} \frac{\left[\gamma_{1}\left(\alpha, \beta x_{i}\right)^{c-2}(c-1)+\gamma_{1}\left(\alpha, \beta x_{i}\right)^{2(c-1)}\right]}{\left[1-\gamma_{1}\left(\alpha, \beta x_{i}\right)^{c}\right]^{2}}+\frac{\partial^{2}\left[\gamma_{1}\left(\alpha, \beta x_{i}\right)\right]}{\partial \beta^{2}} \frac{\left[\gamma_{1}\left(\alpha, \beta x_{i}\right)^{c-1}-\gamma_{1}\left(\alpha, \beta x_{i}\right)^{2 c-1}\right]}{\left[1-\gamma_{1}\left(\alpha, \beta x_{i}\right)^{c}\right]^{2}} \\
y_{\beta a}\left(x_{i}\right) & =\frac{\partial\left[\gamma_{1}\left(\alpha, \beta x_{i}\right)\right] / \partial \beta}{\gamma_{1}\left(\alpha, \beta x_{i}\right)}, \\
y_{\beta b}\left(x_{i}\right)= & \frac{\gamma_{1}\left(\alpha, \beta x_{i}\right)^{c-1}\left\{\partial\left[\gamma_{1}\left(\alpha, \beta x_{i}\right)\right] / \partial \beta\right\}}{1-\gamma_{1}\left(\alpha, \beta x_{i}\right)^{c}}, \\
y_{\beta c}\left(x_{i}\right)= & \frac{\gamma_{1}\left(\alpha, \beta x_{i}\right)^{c-1} \log \left[\gamma_{1}\left(\alpha, \beta x_{i}\right)\right]\left\{\partial\left[\gamma_{1}\left(\alpha, \beta x_{i}\right)\right] / \partial \beta\right\}}{\left[1-\gamma_{1}\left(\alpha, \beta x_{i}\right)^{c}\right]^{2}}, \\
y_{b c}\left(x_{i}\right)= & \frac{\gamma_{1}\left(\alpha, \beta x_{i}\right)^{c} \log \left[\gamma_{1}\left(\alpha, \beta x_{i}\right)\right]}{1-\gamma_{1}\left(\alpha, \beta x_{i}\right)^{c}}, \\
y_{c c}\left(x_{i}\right)= & \frac{\gamma_{1}\left(\alpha, \beta x_{i}\right)^{c} \log \left[\gamma_{1}\left(\alpha, \beta x_{i}\right)\right]^{2}}{\left[1-\gamma_{1}\left(\alpha, \beta x_{i}\right)^{c}\right]^{2}} .
\end{aligned}
$$

\section{References}

Ascher, H., \& Feingold, H. (1984). Repairable systems reliability. New York: Marcel Dekker.

Akinsete, A., Famoye, F., \& Lee, C. (2008). The beta Pareto distribution. Statistics, 42, $547-563$. http://dx.doi.org/10.1080/02331880801983876

Alexander, C., Cordeiro, G. M., Ortega, E. M. M., \& Sarabia, J. M. (2011). Generalized beta-generated distributions. Computational statistics and data analysis. http://dx.doi.org/10.1016/j.csda.2011.11.015

Amoroso, L. (1925). Ricerche intono alla curva dei redditi. Annali di matematica pura ed applicata, 2, $123-159$. http://dx.doi.org/10.1007/BF02409935

Barreto-Souza, W., Santos, A. H. S., \& Cordeiro, G. M. (2010). The beta generalized exponential distribution. Journal of statistical computation and simulation, 80, 159-172. http://dx.doi.org/10.1080/00949650802552402

Bobee, B., \& Ashkar, F. (1991). The gamma family and derived distributions applied in hydrology. Littleton, CO: Water Resources Publications.

Bowley, A. L. (1920). Elements of statistics. New York: C. Scribner's sons.

Cordeiro, G. M., \& Brito, R. (2012). The beta power distribution. Brazilian journal of probability and statistics, 26, 88-112. http://dx.doi.org/10.1214/10-BJPS124

Cordeiro, G. M., \& de Castro, M. (2011). A new family of generalized distributions. Journal of statistical computation and simulation, 81, 883-898. http://dx.doi.org/10.1080/00949650903530745

Cordeiro, G. M., \& Nadarajah, S. (2011). Closed-form expressions for moments of a class of beta generalized distributions. Brazilian journal of probability and statistics, 25, 14-33. http://dx.doi.org/10.1214/09-BJPS109

Cordeiro, G. M., Ortega, E. M. M., \& Silva, G. O. (2010). The exponentiated generalized gamma distribution with application to lifetime data. Journal of statistical computation and simulation, 81, 827-842. http://dx.doi.org/10.1080/00949650903517874

Cox, C. (2008). The generalized f distribution: An umbrella for parametric survival analysis. Statistics in medicine, 27, 4301-4312. http://dx.doi.org/10.1002/sim.3292

de Pascoa, M. A. R., Ortega, E. M. M., \& Cordeiro, G. M. (2011). The Kumaraswamy generalized gamma distribution with application in survival analysis. Statistical methodology, 8, 411-433. http://dx.doi.org/10.1016/j.stamet.2011.04.001

Esteban, J. (1981). Income-share elasticity, density functions and the size distribution of income. Mimeographed manuscript, 
University of Barcelona, Spain.

Eugene, N., Lee, C., \& Famoye, F. (2002). Beta-normal distribution and its applications. Communications in statistics Theory and methods, 31, 175-195. http://dx.doi.org/10.1081/STA-120003130

Gilchrist, W. (2000). Statistical modelling with quantile functions. Boca Raton, FL: CRC Press.

Giorgi, G. M., \& Nadarajah, S. (2010). Bonferroni and Gini indices for various parametric families of distributions. Metron - International journal of statistics, LXVIII, 23-46. ftp://metron.sta.uniroma1.it/RePEc/articoli/2010-1-2.pdf

Gradshteyn, I. S., \& Ryzhik, I. M. (1980). Table of integrals, series, and products. New York: Academic Press.

Jambunathan, M. (1954). Some properties of beta and gamma distributions. The annals of mathematical statistics, 25, 401-405. http://dx.doi.org/10.1214/aoms/1177728800

Jones, M. (2004). Families of distributions arising from distributions of order statistics. Test, 13, 1-43. http://dx.doi.org/10.1007/BF02602999

Lee, C., Famoye, F., \& Olumolade, O. (2007). Beta-Weibull distribution: Some properties and applications to censored data. Journal of modern applied statistical methods, 6, 173-186.

Meeker, W. Q., \& Escobar, L. A. (1998). Statistical methods for reliability data. New York: John Wiley \& Sons.

McDonald, J. (1984). Some generalized functions for the size distribution of income. Econometrica, 52, 647-663. http://www.jstor.org/stable/1913469

Moors, J. J. A. (1986). The meaning of kurtosis: Darlington reexamined. The American Statistician, 40, $283-284$. http://dx.doi.org/10.1080/00031305.1986.10475415

Moors, J. J. A. (1988). A quantile alternative for kurtosis. Journal of the royal statistical society (Series D), 37, 25-32. http://www.jstor.org/stable/2348376

Nadarajah, S. (2008). On the use of the generalized gamma distribution. International journal of electronics, 95, 10291032. http://dx.doi.org/10.1080/00207210802354981

Nadarajah, S., \& Gupta, A. K. (2004). The beta Fréchet distribution. Far east journal of theoretical statistics, 14, 15-24.

Nadarajah, S., \& Gupta, A. K. (2007). A generalized gamma distribution with application to drought data. Mathematics and computers in simulation, 74, 1-7. http://dx.doi.org/10.1016/j.matcom.2006.04.004

Nadarajah, S., \& Kotz, S. (2004). The beta Gumbel distribution. Mathematical problems in engineering, 10, 323-332. http://dx.doi.org/10.1155/S1024123X04403068

Nadarajah, S., \& Kotz, S. (2005). The beta exponential distribution. Reliability engineering $\mathcal{E}$ system safety, 91, 689-697. http://dx.doi.org/10.1016/j.ress.2005.05.008

Nadarajah, S., \& Kotz, S. (2006). The exponentiated type distributions. Acta applicandae mathematicae, 92, 97-111. http://dx.doi.org/10.1007/s10440-006-9055-0

Pescim, R. R., Demétrio, C. G. B., Cordeiro, G. M., Ortega, E. M. M., \& Urbano, M. R. (2006). The beta generalized halfnormal distribution. Computational statistics $\mathcal{E}$ data analysis, 54, 945-957. http://dx.doi.org/10.1016/j.csda.2009.10.007

Sen, P. K., \& Singer, J. M. (1993). Large sample methods in statistics: An introduction with applications. New York: Chapman and Hall.

Stacy, E. W. (1962). A generalization of the gamma distribution. The annals of mathematical statistics, 33, 1187-1192. http://dx.doi.org/10.1214/aoms/1177704481

Steinbrecher, G. (2002). Taylor expansion for inverse error function around origin. Working Paper, University of Craiova, Romania.

Steinbrecher, G., \& Shaw, W. T. (2008). Quantile mechanics. European journal of applied mathematics, 19, 87-112. http://dx.doi.org/10.1017/S0956792508007341

R Development Core Team. (2008). R: A Language and environment for statistical computing. $\mathrm{R}$ foundation for statistical computing, Vienna. ISBN 3-900051-07-0

Yevjevich, V. (1972). Probability and statistics in hydrology. Littleton, CO: Water Resources Publications. 
Table 1. Descriptive statistics for diesel engine data from the USS Halfbeak

\begin{tabular}{cccccccc}
\hline Mean & \multicolumn{2}{c}{ Median Std. Desv. Variance Skewness Kurtosis } & Min & Max \\
\hline 19.3997 & 21.4610 & 5.8165 & 33.8322 & -1.5104 & 4.3305 & 1.3820 & 25.5180 \\
\hline
\end{tabular}

Table 2. MLEs and Goodness-of-fit measures

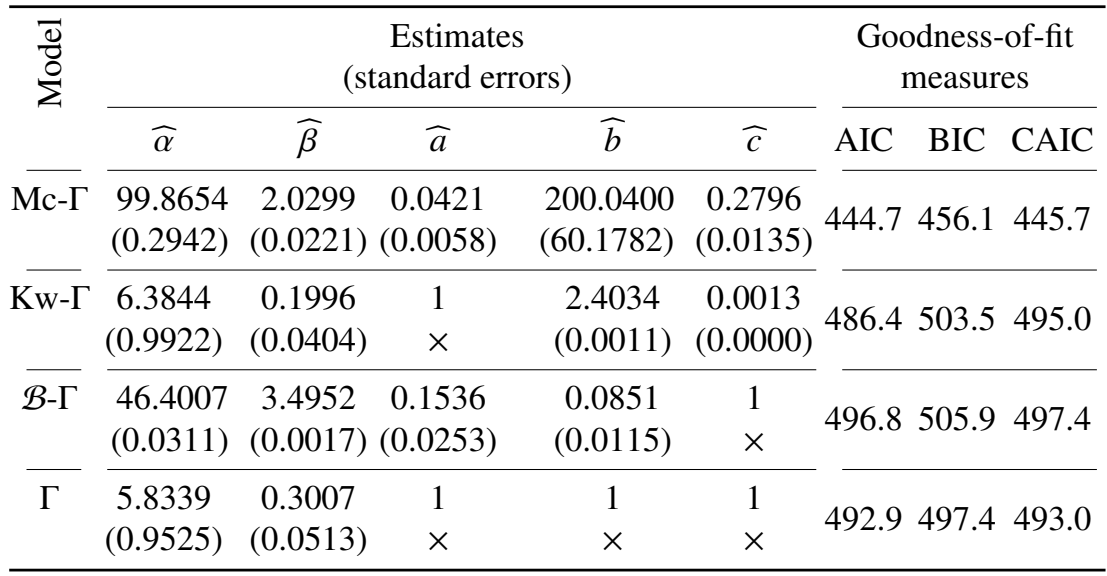

Table 3. LR tests under Mc- $\Gamma$ parameters based on real data

\begin{tabular}{|c|c|c|c|}
\hline Model & Hypotheses & Statistic $\Lambda$ & $p$-value \\
\hline Mc- $\Gamma$ vs Kw- $\Gamma$ & $H_{0}: a=1$ vs $H_{1}: a \neq 1$ & 51.7 & $<0.0001$ \\
\hline Mc- $\Gamma$ vs $\mathcal{B}-\Gamma$ & $H_{0}: c=1$ vs $H_{1}: c \neq 1$ & 54.1 & $<0.0001$ \\
\hline Mc- $\Gamma$ vs $\Gamma$ & $H_{0}: a=b=c=1$ vs $H_{1}: \operatorname{not} H_{0}$ & 54.2 & $<0.0001$ \\
\hline
\end{tabular}

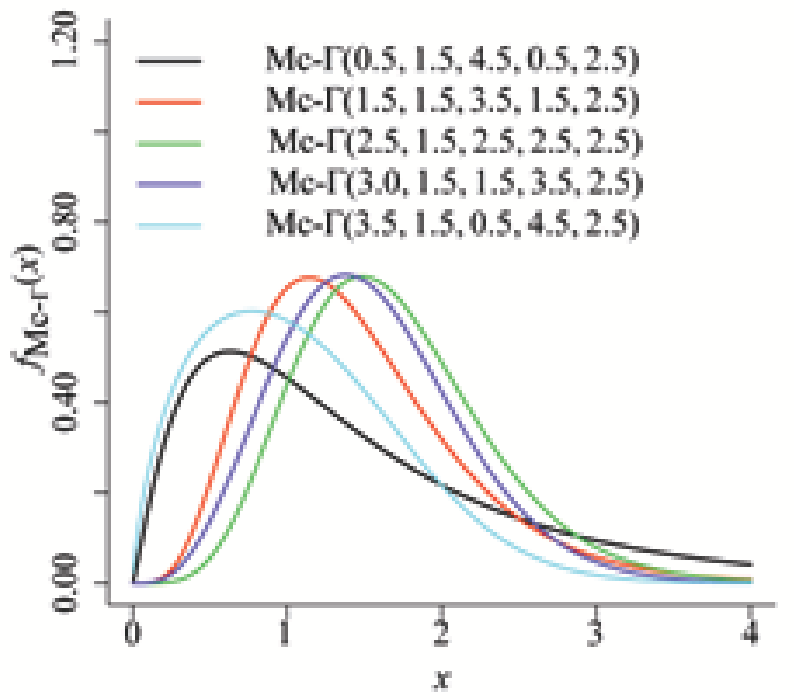

(a)

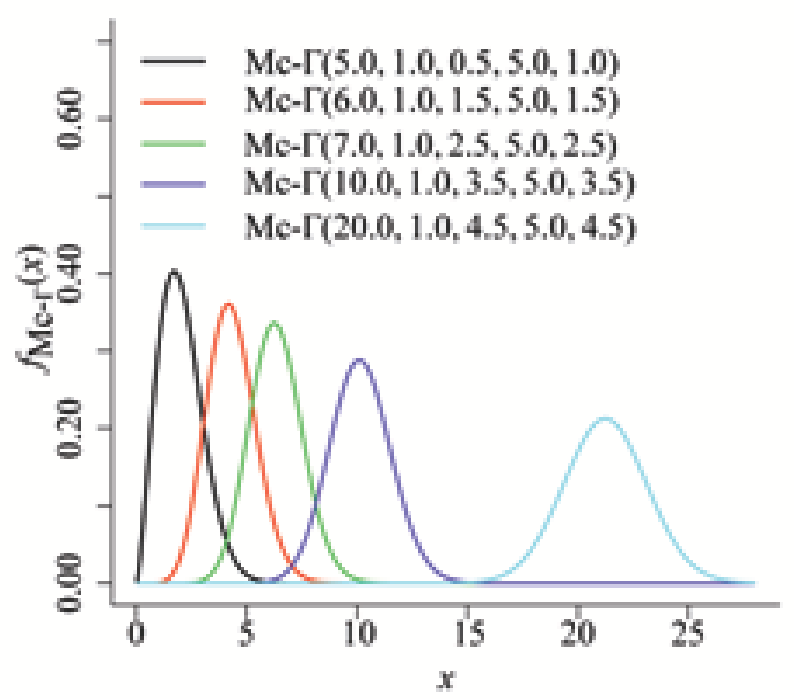

(b)

Figure 1. The Mc- $\Gamma$ density function for some parameter values 


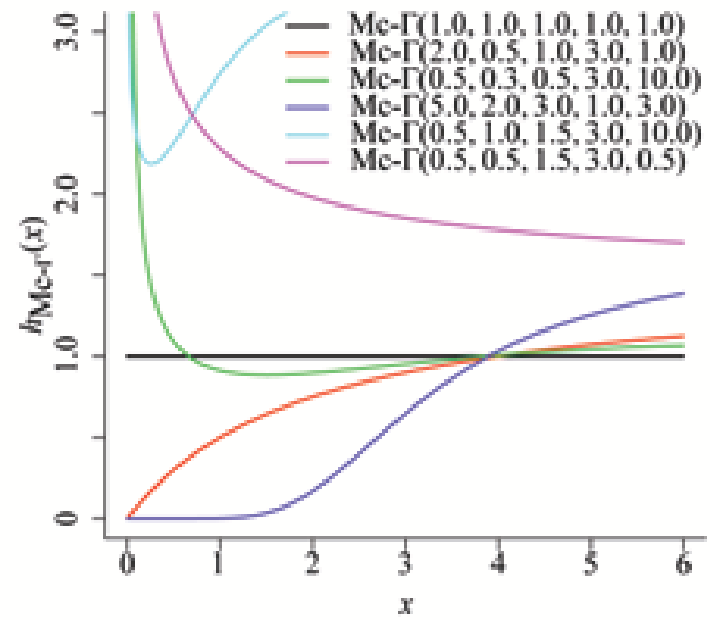

(a)

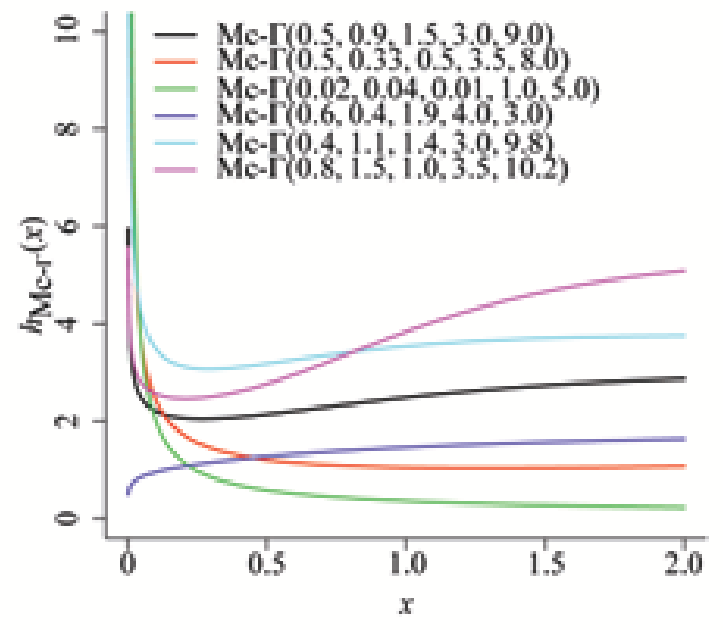

(b)

Figure 2. Plots of the Mc- $\Gamma$ hazard function for some parameter values

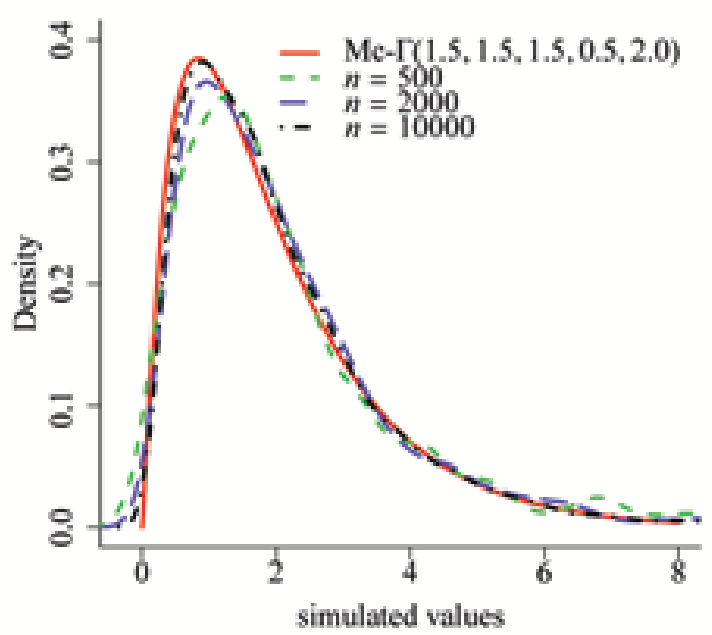

(a)

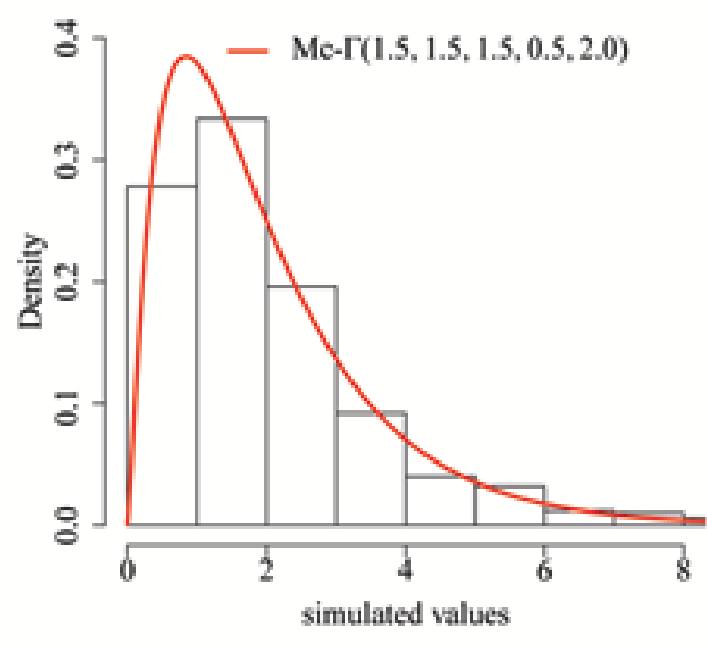

(b)

Figure 3. Approximate density (a) and histogram (b) of the values generated from the theoretical density

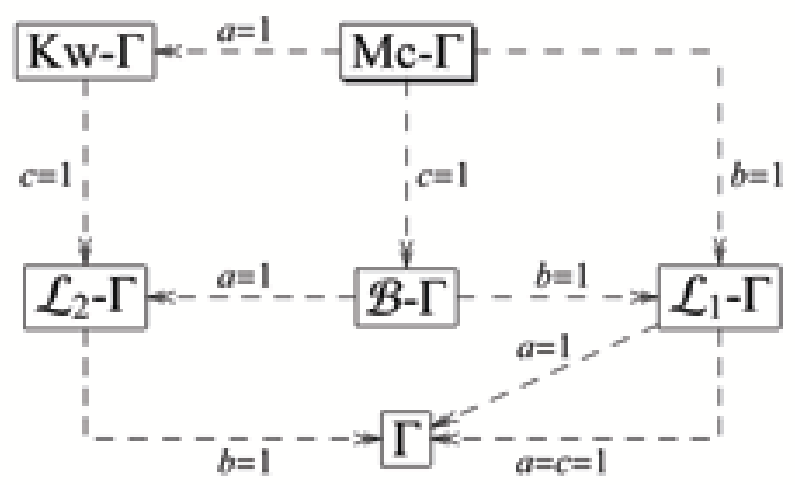

Figure 4. Relationships of the Mc- $\Gamma$ sub-models 


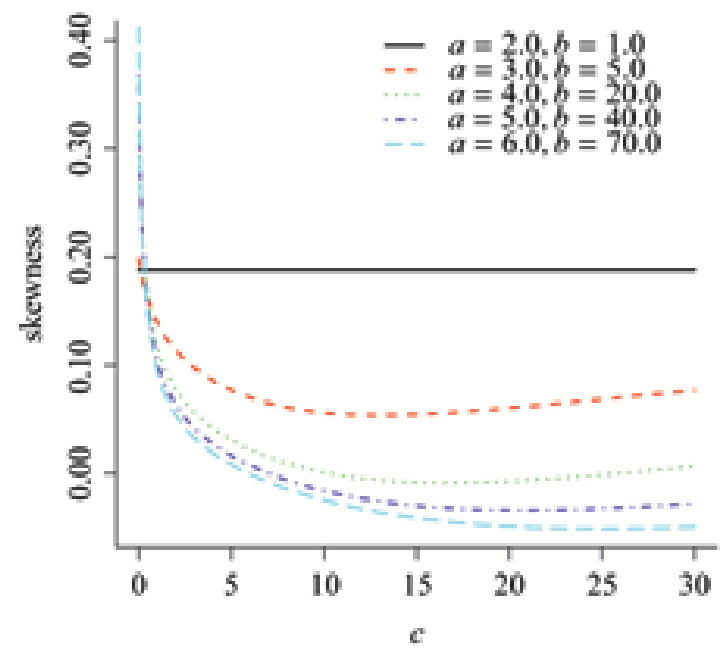

(a)

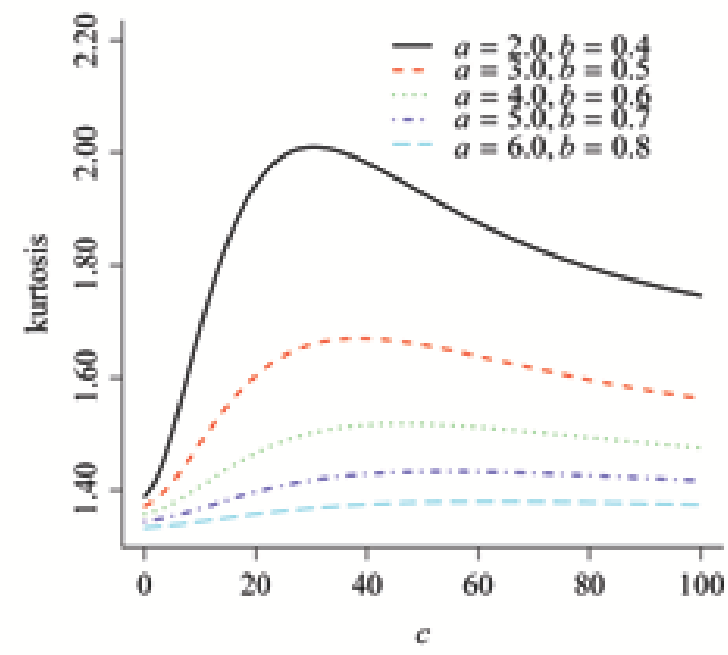

(b)

Figure 5. Skewness and kurtosis of the Mc- $\Gamma$ distribution for different values of $c$ : (a) For $\alpha=1.0$ and $\beta=10$ (skewness), (b) For $\alpha=0.2$ and $\beta=10$ (kurtosis)

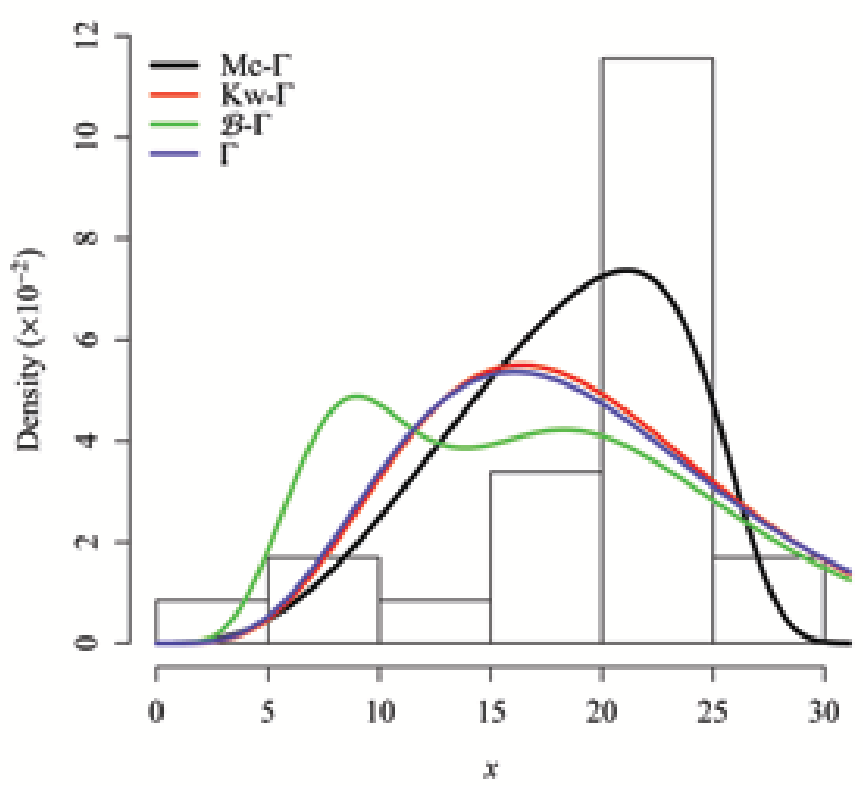

Figure 6. Estimated densities of the $\mathrm{Mc}-\Gamma, \mathrm{Kw}-\Gamma, \mathcal{B}-\Gamma$ and $\Gamma$ models for USS Halfbeak diesel engine data

Notes

Note 1. This author also is a doctoral student at the Federal University of Pernambuco.

Note 2. This result can be obtained in Wolfram Alpha website http://www.wolframalpha.com/ 